\title{
Quantum Mechanics of a Black Hole
}

\author{
Gilad Gour* \\ Racah Institute of Physics, Hebrew University, Givat Ram, Jerusalem 91904, ISRAEL.
}

\begin{abstract}
Beginning with Bekenstein, many authors have considered a uniformly spaced discrete quantum spectrum for black hole horizon area. It is also believed that the huge degeneracy of these area levels corresponds to the notion of black hole entropy. Starting from these two assumptions we here infer the algebra of a Schwarzschild black hole's observables. This algebra then serves as motivation for introducing in the system's Hamiltonian an interaction term. The interaction contains the horizon area operator, which is a number operator, and its canonical conjugate, the phase operator. The Hawking radiation from a Schwarzschild black hole is seen to be a consequence of an area-phase interaction. Using this interaction we have reproduced the semi-classical result for the Hawking radiation power. Furthermore, we show that the initial state of the black hole determines the nature of its development. Thus, a state which is an area eigenstate describes a static eternal black hole, but a coherent state describes a radiating black hole. Hence, it is the observer's initial knowledge or uncertainty about the horizon area which determines the evolution.
\end{abstract}

Typeset using REVTEX

*E-mail: gour@cc.huji.ac.il 


\section{INTRODUCTION}

The first quarter of this century yielded two revolutionary theories, quantum mechanics and general relativity; they have changed drastically the way we think about the physical world. Since then many efforts have been made to unify these theories, and today we are presented with a variety of generic "quantum gravity" theories. Let us mention canonical quantum gravity [1], perturbative quantum gravity [2], superstring and D-brane theory [3], loop (nonperturbative) quantum gravity [1] and others. All these are intricate theories: even comparison of their predictions is a difficult task. In view of the dearth of concrete experimental results bearing on the quantum aspects of gravity, one wonders whether it would not be more to the point to construct a theory which emphasizes some particular gravitational system where circumstances allow a guess as to the general nature of quantum effects. Black holes suggest themselves as a good place to start: they involve strong gravitational fields, have properties reminiscent of localized particles, and results from quantum field theory [principally Hawking's radiance (HR)] suggest that quantum effects are central. Thus an approach to black hole quantization has emerged [5 8] which starts off from hints garnered in classical black hole physics, and proceeds to quantum ideas.

This approach hails back to the realization by Christodoulou [9], Hawking [10] and Penrose and Floyd [11] that transformations of a black hole (BH) generically have an irreversible character. One of its conclusions is that the horizon area tends to grow, a rule which served as motivation for Bekenstein [12] to associate entropy with horizon area $A$ according to (we insert the modern value of the coefficient)

$$
S=A /\left(4 \mathcal{L}_{p}^{2}\right)
$$

$\left(\mathcal{L}_{P}\right.$ and $\mathcal{M}_{P}$ will denote the Planck length and mass, respectively). Christodoulou also showed that there are some special $\mathrm{BH}$ reversible processes which leave $\mathrm{BH}$ area unchanged. This has led Bekenstein [5] to remark that the horizon area of a non-extremal BH behaves as a classical adiabatic invariant (for support of this conjecture see also Bekenstein [13] and Mayo [14]). According to Ehrenfest's principle 15 any classical adiabatic invariant corresponds to a quantum entity with discrete spectrum. Thus Bekenstein conjectured that the spectrum of the horizon area of a (non-extremal) BH should be quantized. Today the idea of a discrete eigenvalue spectrum for the horizon area is also supported by the work of Ashtekhar and others [4].

In support of a conjectured uniform spacing of the area eigenvalues, Bekenstein noted [5,8] that the assimilation of a neutral test particle into a BH increases the horizon area by at least $v \mathcal{L}_{P}^{2}$, where $v$ is a dimensionless constant of order unity. Also Hod [16] have shown independently that the same kind of lower bound on the horizon area increase applies to the $\mathrm{BH}$ assimilation of a charged particle. The fact that the lower bound turns out to be independent of the $\mathrm{BH}$ parameters (mass, charge and angular momentum) is in harmony with a uniformly spaced area spectrum:

$$
a_{n}=v \mathcal{L}_{P}^{2}(n+\eta) ; \quad 0 \leq \eta<1 ; \quad n=0,1,2, \ldots
$$

Here $a_{n}$ is the area eigenvalue. In the present work we shall set $\eta=0$ for simplicity.

The spectrum in Eq. (1.2) together with the classical relation $A=16 \pi M^{2}$ (we use gravitational units in which $G=c=1$ ) implies that for $n \gg 1$ the energy (mass) levels $M_{n}$ 
of an isolated Schwarzschild BH have the form $M_{n}=m_{0} \sqrt{n}$; here $m_{0}=\sqrt{v \hbar / 16 \pi}$. This kind of spectrum is compatible with Bohr's correspondence principle. That is, for large $n$ values the $n \rightarrow n-1$ transition frequency

$$
\omega_{0} \equiv \frac{M_{n}-M_{n-1}}{\hbar}=\frac{v}{32 \pi M}
$$

agrees with the classical BH oscillation frequencies which scale as $M^{-1}$.

The energy $\hbar \omega_{0}$ emitted in the transition $n \rightarrow n-1$ is reminiscent of the Hawking temperature. Hawking [17] has shown that a steady emission of particles to infinity originating in pair creation in the gravitational field follows when a $\mathrm{BH}$ is formed by gravitational collapse. He associated with the $\mathrm{BH}$ a temperature $T_{B H}=\hbar / 8 \pi M$ [compatible with the entropy given in Eq. (1.1)] which characterizes the thermal spectrum of the emitted particles. Note that $T_{B H}$ and $\hbar \omega_{0}$ are of the same order. Hence, the idea that the temperature represent the average energy of the emitted particles is compatible with a mass spectrum $M \sim \sqrt{n}$.

Following Bekenstein's conjecture about the mass spectrum, a number of authors (see full references in [18] and [8]) have obtained the selfsame spectrum from a wide variety of arguments. For example, Vaz and Witten [19 examined the Wheeler-DeWitt equation for a static, eternal Schwarzschild BH in Kuchař-Brown variables, and obtained the mass spectrum $M_{n} \propto \sqrt{n}$. Louko and Mäkelä [20] have proposed a Hamiltonian operator for the Schwarzschild BH, and obtained the same spectrum. Kastrup [21] explained how such a spectrum may be understood in the framework of canonical quantization of a purely gravitational spherically symmetric system. Carlip and Teitelboim [22] used the standard (Euclidean) action principle for the gravitational field to show that the horizon area and the opening angle at the horizon are canonical conjugates. The opening angle is bounded (in the Euclidean sector). This is compatible with the idea that the area operator is represented by a number operator (see Eq. (1.2), since its canonical conjugate is a phase operator [23]. This similarity is further evidence in favor of an equally spaced area spectrum.

The energy levels $M_{n}$ are expected to be degenerate. Denoting the degeneracy by $g(n)$ and identifying $\ln g(n)$ with the entropy given in Eq. (1.1), Bekenstein and Mukhanov [7] found that $v=4 \ln k$ [ $v$ is the dimensionless constant in Eq. (1.2)], or equivalently $g(n)=k^{n}$ where $k=2,3,4, \cdots$ They chose $k=2$ for simplicity; recently Hod [24] has given evidence in favor of the choice $k=3$.

By contrast to the approach just discussed, quantum loop gravity determines a nonuniformly spaced area spectrum. Ashtekhar and Krasnov claim [4 that the area spacing approaches zero exponentially for large quantum numbers. Hence, the area spectrum they propose is directly compatible with the semi-classical prediction by Hawking [17] that the emitted spectrum is smooth. The present paper shows that other features of Hawking radiation emerge naturally from the uniformly spaced area spectrum hypothesis. For example, we show that the rate of change of the mass of a $\mathrm{BH}$ (not near the Planck scale) agrees with that of the semi-classical calculation by Page [25] up to the second order in time. Thus, although the uniformly spaced area spectrum is inimical to the smooth Hawking spectrum, it is not at variance with equally important features of a semi-classical BH.

It is clear that the Hawking temperature is a quantum effect ( $T_{B H}$ is proportional to Planck's constant $\hbar$ ), and a deep understanding of it requires a quantum theory of BHs. In this paper a quantum model for Schwarzschild BHs is proposed. We start from two axioms: 
(a) that the horizon area spectrum is equally spaced, and (b) that the degeneracy of level $n$ is $k^{n}$. Surprisingly, these two axioms enable us to obtain the algebra of the Schwarzschild BH observables, and even the form of the Hamiltonian. The algebra obtained is the algebra of angular momentum with the restriction that the absolute value of the "angular momentum", here called the hyperspin, can have only the values $\left(k^{n}-1\right) / 2$. This last limitation motivates us to introduce an interaction term which allows transitions only between allowed values of the hyperspin.

The $z$-projection of the hyperspin represents the internal degree of freedom of the $\mathrm{BH}$ [this degree of freedom corresponds to the entropy given in Eq. (1.1)]. According to the "no hair" theorems [27], an observer located at large distance from the BH is not able to distinguish between different states of the internal degrees of freedom. Hence, we find it necessary to introduce a horizon projection operator which filters out the hyperspin degree of freedom, but leaves that corresponding to the mass of the BH. This corresponds to the conception that most of the information about the $\mathrm{BH}$ interior is hidden (by the horizon) from an exterior observer.

The Hilbert space which describes the states that the observer at infinity can measure is spanned by area (mass) eigenstates. We show in this paper that the initial state determines the nature of the dynamics of the BH. For example, if the initial state is chosen to be an area eigenstate, it describes a static eternal BH. On the contrary, if the initial state is chosen to be a coherent state,, it reproduces the semi-classical result [25,7] for the Hawking power. That is, it is our initial knowledge (uncertainty) about the horizon area which determines the nature of the dynamics. A BH formed by gravitational collapse generally has uncertain horizon area, and thus it looses mass, whereas an eternal BH, with a definite mass and area, is static.

This paper is organized as follows. In section IIA we construct the hyperspin algebra and characterize the observable Hilbert space. In section III we define the mass operator, and then find the general form of the Hamiltonian of a Schwarzschild BH. We also review the quantum phase problem in general, and then define the phase of a BH. In section IV we discuss the initial states to be considered, and in section $\square$ we use them to compare semiclassical properties of the HR with our model's predictions. Finally, in section VII, we present our summary and conclusions.

\section{BLACK HOLE ALGEBRA}

The classical Schwarzschild BH is characterized by only one parameter, its mass or equivalently its horizon area. Since the horizon area is an adiabatic invariant and is suggested to be quantized (as discussed in the Introduction), we shall focus on it. Hence, the first step should be to investigate the horizon area operator. In this work we shall not construct the area operator from more fundamental quantities [4], even though this might illuminate our perception of the internal structure of the $\mathrm{BH}$. Instead, we shall use the properties of the

\footnotetext{
${ }^{1}$ Coherent states are the ones that most closely imitate the classical results for a simple harmonic oscillator.
} 
area operator which follow from general arguments (see the Introduction). We now turn to construct the algebra.

\section{A. The Algebra}

Let $\mathbf{A}$ represent the horizon area operator (boldface is used everywhere to denote operators). Motivated by the arguments discussed in the Introduction we shall start from the following two axioms:

(1) The eigenvalues of $\mathbf{A}$ are $a_{n}=a_{0} n$ where $n=0,1,2 \ldots, a_{0}=4 \ln k \mathcal{L}_{P}^{2}$ is a positive universal constant, and $k$ is an integer greater then one.

(2) There are exactly $k^{n}$ independent eigenstates of $\mathbf{A}$ with eigenvalue $a_{n}$, i.e. the degeneracy of a state with area $a_{n}$ is $k^{n}$.

From the second axiom it follows that there is at least one more operator which commutes with A. Let $\mathbf{G}$ represent the set of all independent operators which commute with $\mathbf{A}$. Typically,

$$
[\mathbf{A}, \mathbf{G}]=0
$$

and the set $\{\mathbf{A}, \mathbf{G}\}$ of "observables" is maximal in the sense that if $\mathbf{S}$ is another observable which commutes with both $\mathbf{A}$ and $\mathbf{G}$, then $\mathbf{S}=f(\mathbf{A}, \mathbf{G})$ where $f$ is a function of $\mathbf{A}$ and $\mathbf{G}$. We shall call G a "secret" observable since it describes the internal degrees of freedom of the $\mathrm{BH}$.

From the second axiom or from Eq. (2.1) it follows that we can simultaneously diagonalize A and $\mathbf{G}$. Hence we shall denote the states as $|n, m\rangle$ such that

$$
\begin{aligned}
& \mathbf{N}|n, m\rangle=n|n, m\rangle \quad n=0,1,2 \ldots \\
& \mathbf{G}|n, m\rangle=m|n, m\rangle \quad m=1,2, \ldots, k^{n}
\end{aligned}
$$

where $\mathbf{N} \equiv a_{0}^{-1} \mathbf{A}$ is a dimensionless number operator. The eigenvalues $m$ of $\mathbf{G}$ are limited by $n$ since we want a degeneracy of $k^{n}$. We have assumed that $m$ is an integer; if not, instead of $\mathbf{G}$ we shall choose the "secret" operator to be $f(\mathbf{G})$, where $f(m)=1,2, \ldots, k^{n}$ for all the different $k^{n}$ values of $m$.

Now, since $\mathbf{G}$ has a discrete spectrum $m=1,2, \ldots, k^{n}$, we shall introduce the annihilation operator $\mathbf{g}$ and the creation operator $\mathbf{g}^{\dagger}$ corresponding to $\mathbf{G}$ :

$$
[\mathrm{G}, \mathrm{g}]=-\mathrm{g} \quad\left[\mathrm{G}, \mathrm{g}^{\dagger}\right]=\mathrm{g}^{\dagger}
$$

As we shall see shortly, under certain conditions $\mathbf{g}$ and $\mathbf{g}^{\dagger}$ have to satisfy a certain commutation relation. In order to single out an algebra, we have to demand that $\left[\mathbf{g}, \mathbf{g}^{\dagger}\right]$ be linear in G. In order to derive this commutation rule we shall first guess some of the properties of $\mathbf{g}$ and $\mathbf{g}^{\dagger}$ from physical arguments. First we do not want $\mathbf{g}$ and $\mathbf{g}^{\dagger}$ to change $n$ when they act on states with a definite area eigenvalue $n$. This means that transitions among the internal degrees of freedom $\left(m \rightarrow m^{\prime}\right)$ do not have to change the area of the BH. Thus, the area operator $\mathbf{N}$ commutes with $\mathbf{g}$ and $\mathbf{g}^{\dagger}$.

Next, from Eq. (2.3) we have by analogy with the harmonic oscillator

$$
\mathbf{g}^{\dagger}|n, m\rangle \propto|n, m+1\rangle .
$$


Therefore $\mathbf{g}^{\dagger}\left|n, k^{n}\right\rangle=0$ and thus also $\mathbf{g g}^{\dagger}\left|n, k^{n}\right\rangle=0$. It is clear from Eq. (2.3) that [G, $\left.\mathbf{g g}^{\dagger}\right]=$ 0 and also that $\left[\mathbf{N}, \mathbf{g g}^{\dagger}\right]=0$. But the set $(\mathbf{N}, \mathbf{G})$ is maximal and thus, since $\mathbf{g g}^{\dagger}$ is an observable, $\mathbf{g g}^{\dagger}=h_{1}(\mathbf{N}, \mathbf{G})$ where $h_{1}$ is a function of $\mathbf{A}$ and $\mathbf{G}$. Hence, from the fact that $\mathbf{g g}^{\dagger}\left|n, k^{n}\right\rangle=0$ we find that $h_{1}\left(n, k^{n}\right)=0$. Similarly we find that

$$
\mathbf{g}^{\dagger} \mathbf{g}|n, 1\rangle=0
$$

where $\mathbf{g}^{\dagger} \mathbf{g}=h_{2}(\mathbf{N}, \mathbf{G})$. It follows from Eq. (2.5) that $h_{2}(n, 1)=0$. Collecting all of these facts we find that $\mathbf{g g}^{\dagger}$ and $\mathbf{g}^{\dagger} \mathbf{g}$ can be written uniquely as

$$
\begin{aligned}
& \mathbf{g g}^{\dagger}=u(\mathbf{N}) \mathbf{G}\left(k^{\mathbf{N}}-\mathbf{G}\right) \\
& \mathbf{g}^{\dagger} \mathbf{g}=u(\mathbf{N})\left(k^{\mathbf{N}}-\mathbf{G}+1\right)(\mathbf{G}-1),
\end{aligned}
$$

where $u(\mathbf{N})$ is some function of $\mathbf{N}$. In Appendix A we show that $u(\mathbf{N})=1$. Thus, we settle on the commutation relation

$$
\left[\mathbf{g}, \mathbf{g}^{\dagger}\right]=k^{\mathbf{N}}-2 \mathbf{G}+1
$$

which is linear in G. Eq. (2.7) and Eq. (2.3) seem to imply that the set of operators $\left\{\mathbf{G}, \mathbf{g}, \mathbf{g}^{\dagger}\right\}$ plus the identity element span a 4 -dimensional Lie algebra which constitutes a central extension of the rotation group. The representations are supposed to be such that the maximal value of the third component is just $k^{n}$. The type of the central extension is exhibited in Eq. (2.7) where the term $\left(k^{n}+1\right) / 2$ is the central charge (a multiple of the identity). However, as we shall see later, we can eliminate the central charge from Eq. (2.7) by a redefinition of the third generator $\mathbf{G} \rightarrow \mathbf{G}-\left(k^{n}+1\right) / 2$. Furthermore, we shall make it clear under what circumstances the commutation rule (2.7) is consistent with the required spectrum of $\mathbf{N}$ and $\mathbf{G}$.

\section{The Matrix Elements}

We turn now to calculate the matrix elements of the operators in the algebra. We chose the orthonormal set of states $\{|n, m\rangle\}_{m=1,2, \ldots, k^{n}}^{n=0,1,2, \ldots}$ as a basis. Thus we have

$$
\begin{aligned}
\left\langle n^{\prime} m^{\prime}|\mathbf{N}| n, m\right\rangle & =n \delta_{n, n^{\prime}} \delta_{m, m^{\prime}} \\
\left\langle n^{\prime} m^{\prime}|\mathbf{G}| n, m\right\rangle & =m \delta_{n, n^{\prime}} \delta_{m, m^{\prime}} .
\end{aligned}
$$

Now, from (2.4) it follows that $\mathbf{g}^{\dagger}|n, m\rangle=C_{n m}^{+}|n, m+1\rangle$ where

$$
\left|C_{n m}^{+}\right|^{2}=\left\langle n m\left|\mathbf{g g}^{\dagger}\right| n, m\right\rangle=\left\langle n m\left|\mathbf{G}\left(k^{\mathbf{N}}-\mathbf{G}\right)\right| n, m\right\rangle=m\left(k^{n}-m\right) .
$$

In a similar fashion $\mathbf{g}|n, m\rangle=C_{n m}^{-}|n, m-1\rangle$ were

$$
\begin{aligned}
\left|C_{n m}^{-}\right|^{2}=\left\langle n m\left|\mathbf{g}^{\dagger} \mathbf{g}\right| n, m\right\rangle & =\left\langle n m\left|\left(k^{\mathbf{N}}-\mathbf{G}+1\right)(\mathbf{G}-1)\right| n, m\right\rangle \\
& =\left(k^{n}-m+1\right)(m-1)
\end{aligned}
$$

and thus we finally obtain for the matrix elements of $\mathbf{g}$ and $\mathbf{g}^{\dagger}$ :

$$
\begin{aligned}
\left\langle n^{\prime} m^{\prime}\left|\mathbf{g}^{\dagger}\right| n, m\right\rangle & =\left(m\left(k^{n}-m\right)\right)^{1 / 2} \delta_{n, n^{\prime}} \delta_{m^{\prime}, m+1} \\
\left\langle n^{\prime} m^{\prime}|\mathbf{g}| n, m\right\rangle & =\left(\left(k^{n}-m+1\right)(m-1)\right)^{1 / 2} \delta_{n, n^{\prime}} \delta_{m^{\prime}, m-1},
\end{aligned}
$$

where for simplicity we have chosen $C_{n m}^{+}$and $C_{n m}^{-}$to be a positive real numbers. These matrix elements are reminiscent of the matrix elements of $J_{-} \equiv J_{x}-i J_{y}$ and $J_{-} \equiv J_{x}+i J_{y}$ in the algebra of angular momentum. As we shall see shortly, this is not accidental. 


\section{The Hyperspin of a Black Hole}

Using Eq. (2.6) we can find a relation between $\mathbf{N}, \mathbf{g}$ and $\mathbf{g}^{\dagger}$

$$
k^{2 \mathbf{N}}=\left[\mathbf{g}, \mathbf{g}^{\dagger}\right]^{2}+2\left\{\mathbf{g}, \mathbf{g}^{\dagger}\right\}+1
$$

where the symbol $\{$,$\} indicates an anticommutator. It is not clear a priori that building \mathbf{N}$ from $\mathbf{g}$ and $\mathbf{g}^{\dagger}$ in this way is consistent with the required spectrum of $\mathbf{N}$. In order to clarify this point let us first define three observables:

$$
\begin{aligned}
\mathbf{S}_{\mathbf{1}} & \equiv \frac{1}{2}\left(\mathbf{g}+\mathbf{g}^{\dagger}\right) \\
\mathbf{S}_{\mathbf{2}} & \equiv \frac{i}{2}\left(\mathbf{g}-\mathbf{g}^{\dagger}\right) \\
\mathbf{S}_{\mathbf{3}} & \equiv \mathbf{G}-\frac{1}{2} k^{\mathbf{N}}-\frac{1}{2} .
\end{aligned}
$$

Using the commutation relations between $\mathbf{g}, \mathbf{g}^{\dagger}, \mathbf{N}$ and $\mathbf{G}$ we notice that the operators defined in (2.14) satisfy the standard commutation rules of an angular momentum, that is

$$
\left[\mathbf{S}_{i}, \mathbf{S}_{j}\right]=i \varepsilon_{i j k} \mathbf{S}_{k}
$$

where $\varepsilon_{i j k}$ is the standard antisymmetric tensor. This angular momentum algebra is not surprising for an algebra with three generators. As we shall see below, the requirement that $\mathbf{N}$ is a number operator complicates the algebra.

Let us now rewrite Eq. (2.13) in terms of the angular momentum operators:

$$
k^{2 \mathbf{N}}=4 \mathbf{S}^{2}+1
$$

where $\mathbf{S}^{2}=\mathbf{S}_{1}^{2}+\mathbf{S}_{2}^{2}+\mathbf{S}_{3}^{2}=s(s+1)$ ( $s$ is a half integer). It is clear from Eq. (2.16) and the definition of $\mathbf{S}_{\mathbf{3}}$, that a simultaneous eigenstate of $\mathbf{S}^{2}$ and $\mathbf{S}_{\mathbf{3}}$, which is denoted by $\left|s, m_{3}\right\rangle$, is also a simultaneous eigenstate of $\mathbf{N}$ and $\mathbf{G}$. Explicitly, $\left|s, m_{3}\right\rangle=|n, m\rangle$ for

$$
m=s+m_{3}+1 \quad \text { and } \quad k^{n}=2 s+1 .
$$

Two features follow from this relation. Firstly, the modulus of the angular momentum (the "hyperspin" of the $\mathrm{BH}$ ) is a true observable, whereas its z-projection represents the hidden degrees of freedom of the BH. Secondly, we can see that $n$ may be an integer, but it may also be $\log _{k}(2 s+1)$ where $s=0, \frac{1}{2}, 1, \frac{3}{2}, 2, \ldots$. Thus, the Hilbert space $\mathcal{H}$ spanned by the set $\{|n, m\rangle\}$ for an integer $n$ is a subspace of the "full" Hilbert space $\mathcal{H}_{\mathrm{f}}$ spanned by the set $\left\{\left|s, m_{3}\right\rangle\right\}$. Since we have obtained the angular momentum operators, one can build the algebra in the opposite direction (starting with the three angular momentum operators) and ask, what is the physical reason that a BH state is restricted to a proper subspace of $\mathcal{H}_{\mathrm{f}}$ ? Why can it not have general values of $s$, but only those of the form $\left(k^{n}-1\right) / 2$ ?

These questions motivate us to enter into the dynamics, and define the Hamiltonian. It is physically clear that transitions between states with spin of the form $\left(k^{n}-1\right) / 2$ and states which are not of the same form are forbidden. Before starting to construct the Hamiltonian, let us introduce the Hilbert space of an observer who is located very far a way from the $\mathrm{BH}$. 


\section{B. The Observable Hilbert Space}

An observer far away from a Schwarzschild BH is able to measure only the mass of the BH. "No hair" theorems [27] suggest that this is the sole parameter characterizing the BH. On the other hand, the states in Eq. (2.2) are characterized by the two parameters $n$ and $m$. This raises the question, how can we harmonize between our states and "no hair" theorems? In other words, how to include the Israel-Carter conjecture that "black holes have no hair" in the Hilbert space which is spanned by the degenerate area eigenstates?

At first sight, a description of BHs by means of mixed states with entropy $S=A / 4 \mathcal{L}_{p}^{2}$ instead of pure states (zero entropy) seems to give the answer to the question above. Denoting the density matrix by $\rho_{n}$ for each area eigenvalue $n$, we find that

$$
\rho_{n} \equiv \frac{1}{k^{n}} \sum_{m=1}^{k^{n}}|n, m\rangle\langle n, m|
$$

and thus the entropy $S \equiv-\operatorname{Tr} \rho_{n} \ln \rho_{n}=A / 4 \mathcal{L}_{p}^{2}$. This agrees with Hawking's principle of ignorance [29]: "the surface emits with equal probability all configurations of particles compatible with the observers limited knowledge". However, the problem with Eq. (2.18) is that it suggests that the observer is able to identify $\left|n, m_{1}\right\rangle$ as a different state from $\left|n, m_{2}\right\rangle$ where $m_{1} \neq m_{2}$. Thus, Eq. (2.18) is not compatible with "no hair" theorems. But without a density matrix, how to attribute a $\mathrm{BH}$ an entropy $S=A / 4 \mathcal{L}_{p}^{2}$ ? In order to resolve this issue we introduce the concept of a secret observable.

Motivated by "no hair" theorems we claim that the observer is not able to distinguish between different states with the same area eigenvalue. Hence there is a subspace of $\mathcal{H}$ which represents the observable Hilbert space. In this subspace for each area eigenvalue there is only one area eigenstate, i.e. the area operator is not degenerate in the observable subspace. Now, each area eigenstate in the observable subspace is a linear superposition of the states $\{|n, m\rangle\}_{m=1,2, \ldots, k^{n}}$, that is

$$
|n\rangle_{o b} \equiv \sum_{m=1}^{k^{n}} d_{m}^{n}|n, m\rangle \quad \text { where } \quad \sum_{m=1}^{k^{n}}\left|d_{m}^{n}\right|^{2}=1 .
$$

At first sight Eq. (2.19) implies that an observer at spatial infinity would have to attribute the $\mathrm{BH}$ a vanishing (Bekenstein) entropy. However, the observer is not able to measure the coefficient $d_{m}^{n}$, and this lack of information corresponds to the Bekenstein entropy. The observable Schwarzschild Hilbert space $\mathcal{H}_{o b}$ is then defined as the subspace of $\mathcal{H}$ which is spanned by the states defined in Eq. (2.19):

$$
\mathcal{H}_{o b} \equiv \operatorname{span}\left\{|n\rangle_{o b}\right\}
$$

We shall now explain why the above definitions are relevant to the question discussed at the beginning of this section. The horizon splits the space in two parts. All the physical processes which occur in the interior part of the $\mathrm{BH}$ spacetime can be viewed from the exterior only through a change in the mass of the BH. Hence, physics inside the horizon should be described in terms of the states $|n, m\rangle \in \mathcal{H}$, whereas the physics as it is viewed at infinity should be described in terms of the observable states $|n\rangle_{o b} \in \mathcal{H}_{o b}$. That is, the horizon filters out the knowledge about the internal degrees of freedom. 
We shall define the horizon projection operator:

$$
\mathbf{h} \equiv \sum_{n=0}^{\infty}|n\rangle\langle n|
$$

where from now on $|n\rangle \equiv|n\rangle_{o b}$. Note that the horizon projection operator is the unit operator in the observable subspace. The projection operator represents the horizon in which all the parameters describing the $\mathrm{BH}$ are hidden apart from its mass. That is, each state $|\psi\rangle$ in $\mathcal{H}$ and each observable $\mathbf{B}$ in $\mathcal{H}$ are reduced to

$$
\begin{gathered}
|\psi\rangle \longrightarrow|\psi\rangle_{o b} \equiv \mathbf{h}|\psi\rangle \\
\mathbf{B} \longrightarrow \mathbf{B}_{o b} \equiv \mathbf{h B h}
\end{gathered}
$$

when a physical measurement is made by an observer located at large distance from the BH.

Eq. (2.22) is a manifestation of the concept of a secret observable. It can be regarded as a quantum version of the "no hair" theorems because it implies that an observer at infinity cannot predict the internal state of the $\mathrm{BH}$ apart from its mass, or equivalently its area (the horizon projection operator erases the knowledge about $\mathbf{G}$ ). There are, indeed, two systems involved. One is the "real" physical system of the BH (without the horizon filter) which is described by the mixed state given in Eq. (2.18). Due to the internal degrees of freedom the system has an entropy $S=A / 4 \mathcal{L}_{p}^{2}$. The other system is a small 'slice' of the real physical system (2.20), and only this 'slice' is accessible to the observer at infinity. One can view it as if the observer is wearing a pair of glasses that filter out all but one color (that is, the observer state $\left.|n\rangle_{o b}\right)$. We now turn to find the form of the observable Hamiltonian.

\section{THE HAMILTONIAN}

There are two properties which an observer located at large distance from the $\mathrm{BH}$ can measure: the mass of the $\mathrm{BH}$ (including the energy in the medium between the $\mathrm{BH}$ and the observer) and the energy flux (if there is one) coming from the BH. Hence, we shall start this section by defining an operator in the algebra which represents the mass of the $\mathrm{BH}$.

\section{A. The Mass Operator}

In section $[\mathrm{II}$ the set $(\mathbf{A}, \mathbf{G})$ had been chosen to be maximal in the sense that if $\mathbf{S}$ is another observable which commutes with both $\mathbf{A}$ and $\mathbf{G}$, then $\mathbf{S}$ is a function of $\mathbf{A}$ and $\mathbf{G}$. Now, in the observable Hilbert space, the above argument reduces to the following one: if $\mathbf{S}$ is an observable in $\mathcal{H}_{o b}$ and $[\mathbf{A}, \mathbf{S}]=0$ then $\mathbf{S}$ is a function of $\mathbf{A}$. We shall assume that the mass operator $\mathbf{M}$ which represents the $\mathrm{BH}$ mass commutes with $\mathbf{A}$. That is, $\mathbf{M}$ is a function of $\mathbf{A}$.

Classically, the mass of the $\mathrm{BH}$ can be obtained from its area by the well known relation $A=16 \pi M^{2}$. We shall assume that to a good approximation this relation also holds true for the operators, and hence the mass operator can be written as

$$
\mathbf{M} \equiv \sqrt{\frac{\mathbf{A}}{16 \pi}}(1+\epsilon(\mathbf{A}))
$$


where the eigenvalues of $\mathbf{M}$ constitute the mass spectrum of the $\mathrm{BH}$. The dimensionless function $\epsilon(\mathbf{A})$ must approaches zero in the limit of a massive $\mathrm{BH}$ in order to reproduce the classical relation $A=16 \pi M^{2}$. Now, since $\epsilon(\mathbf{A})$ is a dimensionless function of $\mathbf{A}$ it can be approximated, to first order, by some power of $\mathcal{L}_{P}^{2} / \mathbf{A}$. Hence, its contribution to Eq. (3.1) may be significant only for BHs of the order of the Planck mass. We shall be interested in $\mathrm{BHs}$ not near the Planck mass, and thus we redefine the mass operator as

$$
\mathbf{M} \equiv \sqrt{\frac{\mathbf{A}}{16 \pi}}
$$

\section{B. General Form of the Hamiltonian}

We shall now try to find the form of the Hamiltonian. If we assume that it is equal to $\mathbf{M}$, we can not understand why the hyperspin of the $\mathrm{BH}$ can have eigenvalues only of the form $\left(k^{n}-1\right) / 2$. Moreover, this Hamiltonian describes a static system which clashes with the existence of the HR.

Let us now list the demands from the correct Hamiltonian:

(1) It should be a Hermitian operator. (2) For a very large mass the Hamiltonian of the system should be equal to the mass of the black hole. (3) It should allow transitions only between states with hyperspins $s$ and $s^{\prime}$ which satisfy the condition $s^{\prime}=s \pm k^{n} / 2$ for some integer $n$. (4) It does not contain G. The last demand arises because in the observer's Hilbert space the area operator is not degenerate (see section [IB). From these requirements it follows that we can write the observable Hamiltonian in the form

$$
\mathbf{H}=\mathbf{M}+\mathbf{V}
$$

where V describes an "interaction term" which approaches zero as the BH mass becomes very large.

In section II we have defined three Hilbert spaces. The first one, is the "full" Hilbert space $\mathcal{H}_{f}$ which is spanned by the set of hyperspin states $\left\{\left|s, m_{3}\right\rangle\right\}$. The second is a subspace $\mathcal{H}$ of the full Hilbert space which is spanned by the set of states $\{|n, m\rangle\}$ where $n$ and $m$ are given in Eq. (2.17). The third Hilbert space is a subspace $\mathcal{H}_{o b}$ of $\mathcal{H}$ which is spanned by the observable states defined in Eq. (2.19). In section IIB we have explained why, in the eyes of an observer at infinity, $\mathcal{H}_{o b}$ is the relevant space to work with. The question remains, why we did have to define $\mathcal{H}_{o b}$ as a subspace of $\mathcal{H}$ (with area spectrum $a_{n}=a_{0} n$ ) and not of $\mathcal{H}_{f}$ (with area spectrum $\left.a_{s}=\log _{k}(2 s+1)\right)$ ? Equivalently, why should the area eigenvalues be equally spaced? The answer to the questions above lies in the third requirement on H. Let $|s\rangle$ and $\left|s^{\prime}\right\rangle$ be some states in $\mathcal{H}_{f}$ with hyperspin $s$ and $s^{\prime}$ respectively. Then, by implementing the third requirement on $\mathbf{H}$ we find that $\left\langle s^{\prime}|\mathbf{H}| s\right\rangle=0$ unless $s=s^{\prime} \pm k^{n^{*}} / 2$ where $n^{*}$ is an integer. Thus, if the initial state of the $\mathrm{BH},|s\rangle$, belongs also to $\mathcal{H}$ (that is $\left.s=\left(k^{n}-1\right) / 2\right)$, then $\left\langle s^{\prime}|\mathbf{H}| s\right\rangle=0$ unless $\left|s^{\prime}\right\rangle \in \mathcal{H}$; hence the evolution of the system takes place in a subspace $\mathcal{H}$ of $\mathcal{H}_{f}$.

On the other hand, if the initial state $|s\rangle$ does not belong to $\mathcal{H}$ then $\left\langle s^{\prime}|\mathbf{H}| s\right\rangle=0$ for any $\left|s^{\prime}\right\rangle \in \mathcal{H}$ so that $\mathcal{H}$ does not play any role in the evolution of the $\mathrm{BH}$. In this case, the area eigenvalue of the initial state $|s\rangle$ is $a_{s}=\log _{k}(2 s+1)$ which is not in general an integer, but 
we can still write $a_{s}=n+\eta$ where $n$ is an integer and $0<\eta<1$. This eigenvalue is still consistent with Eq. (1.2) given in the Introduction for the area eigenvalues. Hence, we shall define a subspace $\mathcal{H}_{\eta}$ of $\mathcal{H}_{f}$ as follows:

$$
\mathcal{H}_{\eta} \equiv \operatorname{span}\left\{\left|s_{n} \equiv n+\eta, m_{3}\right\rangle\right\}
$$

where $n=0,1,2, \ldots$ and $m_{3}=-s_{n} / 2,-s_{n} / 2+1, \ldots, s_{n} / 2$. In the same manner, the third requirement on $\mathbf{H}$ implies that the time evolution of the $\mathrm{BH}$ takes place in $\mathcal{H}_{\eta}$. Hence, it is clear now that the third demand on the Hamiltonian is consistent with the spectrum of Eq. (1.2). From now on, for simplicity, we choose $\eta=0$ (note that $\mathcal{H}=\mathcal{H}_{\eta=0}$ ).

We have explained why the third demand on $\mathbf{H}$ is necessary in order to shift attention from the full Hilbert space to the subspace $\mathcal{H}$ which yields the degeneracy $k^{n}$ for the number operator $\mathbf{N}$. We shall now be interested in the kind of interaction term $\mathbf{V}$ which implements the third requirement.

We introduce raising and lowering operators $\mathbf{E}^{\dagger}$ and $\mathbf{E}$ of $\mathbf{A}$, respectively, which we shall define explicitly later. These operators must satisfy the following commutation relation

$$
\left[\mathbf{A}, \mathbf{E}^{\dagger}\right]=\mathbf{E}^{\dagger} \text { and }[\mathbf{A}, \mathbf{E}]=-\mathbf{E}
$$

From the third requirement on $\mathbf{H}$ it is quite clear that $\mathbf{V}$ contains $\mathbf{E}^{\dagger}$ and $\mathbf{E}$ since $(\mathbf{E})^{n}$, for example, allows only transitions between states with hyperspins $s$ and $s-k^{n} / 2$. In addition, we are not interested in products of the form $\mathbf{E}^{\dagger} \mathbf{E}$ since they keep $\mathbf{A}$ unchanged (that is, $\left[\mathbf{A}, \mathbf{E}^{\dagger} \mathbf{E}\right]=0$ ). Combining all of this with the hermiticity of $\mathbf{V}$ we find that $\mathbf{V}$ can be written as

$$
\mathbf{V}=h(\mathbf{A}, \mathbf{E})+h^{*}\left(\mathbf{A}, \mathbf{E}^{\dagger}\right)
$$

where $h$ is a complex function of $\mathbf{A}$ and $\mathbf{E}$.

Now we shall expand the function $h$ as a power series in $\mathbf{E}$. That is, the first term is proportional to $\mathbf{E}$, the second one to $(\mathbf{E})^{2}$ etc. It is reasonable to assume that in a perturbative theory the transitions $n \rightarrow n \pm 1$ have more weight than higher order transitions (such as $n \rightarrow n \pm 2$ ). Hence we shall assume that up to a given approximation, $h$ is linear in $\mathbf{E}$. Later on, we shall justify this assumption by a comparison with the HR for a massive $\mathrm{BH}$. But note that despite the linearization of $h$, the time-evolution operator, which is an exponential function of $\mathbf{E}$ and $\mathbf{E}^{\dagger}$, contains all orders of these operators. We can thus write the "interaction" term as

$$
\mathbf{V}=f(\mathbf{A}) \mathbf{E}+\mathbf{E}^{\dagger} f^{*}(\mathbf{A})
$$

where $f$ is some function of $\mathbf{A}$. If we normalize the raising and lowering operators to have expectation values of the order of unity (as we shall see shortly), it follows from the second requirement on the Hamiltonian that the function $f(n)$ approaches zero as the parameter $n(n=\langle\mathbf{A}\rangle)$ approaches infinity. The question is how fast $f(n)$ approaches zero. We shall give here a motivation that $f(n) \sim 1 / \sqrt{n}$ for large $n$, and in section $\mathbb{V}$ we shall estimate the function $f(n)$ in a more deductive manner.

It was shown by Kastrup [18] that there is a small correction to the BH entropy calculated in canonical ensemble of the order of the logarithm of the area. Likewise, we [30] have 
obtained a logarithmic correction to the entropy by using the grand-canonical approach (we have shown that the grand-canonical approach is also compatible with the HR). Padmanabhan [31], as well as Kim, Lee and Ji [32], have shown that for a small number of particles in the background of the Schwarzschild metric, (or if the system obeys Boltzmann statistics), the entropy is proportional to the logarithm of the area. This implies that there might be a correction to the entropy of the order of the logarithm of $A$. Motivated by the above arguments we shall write the entropy as:

$$
S=\frac{1}{4 \mathcal{L}_{P}^{2}} A+b \ln (d A)
$$

where $b$ and $d$ are constants. Thus we find a small correction to the energy $U$ of the BH:

$$
d U=T_{B H} d S=d M+\frac{b^{\prime}}{M^{2}} d M
$$

where $b^{\prime}$ is some constant. Note that the correction to Hawking temperature $T_{B H}$, if there is one, is absorbed in $b^{\prime}$. Hence, the correction to the energy $U$ is proportional to the reciprocal of $M$. This is one reason for guessing that the function $f(\mathbf{A})$ in Eq. (3.7) is proportional to $1 / \sqrt{\mathbf{A}}$ for large $\langle\mathbf{A}\rangle$ values. As we have mentioned earlier, we shall later derive this result in a more rigorous way. We now turn to define the operators $\mathbf{E}$ and $\mathbf{E}^{\dagger}$ explicitly.

The most general form of a raising operator $\mathbf{a}^{\dagger}$ in the Hilbert space $\mathcal{H}$ is given by

$$
\mathbf{a}^{\dagger}=\sum_{n=0}^{\infty} \sum_{m_{1}=1}^{k^{n}} \sum_{m_{2}=1}^{k^{n+1}} C_{m_{1} m_{2}}^{n}\left|n+1, m_{2}\right\rangle\left\langle n, m_{1}\right|
$$

where $C_{m_{1} m_{2}}^{n}$ are complex numbers. The lowering operator a is defined by taking the Hermitian conjugate of Eq. (3.10). Now, according to Eq. (2.22), in the observable Hilbert space $\mathcal{H}_{o b}$, the raising operator $\mathbf{a}^{\dagger}$ transforms to $\mathbf{h} \mathbf{a}^{\dagger} \mathbf{h}$. That is, the raising operator in $\mathcal{H}_{o b}$ is defined in terms of the observable states $|n\rangle$ instead of Eq. (3.10). Hence, we are able to define $\mathbf{E}$ and $\mathbf{E}^{\dagger}$ as the Susskind-Glogower operators [33,

$$
\mathbf{E}=\sum_{n=0}^{\infty}|n\rangle\langle n+1| \text { and } \mathbf{E}^{\dagger}=\sum_{n=0}^{\infty}|n+1\rangle\langle n| .
$$

It is clear from the definition that these operators satisfy relation (3.5). We can define also the standard creation and annihilation operators, $\mathbf{a}$ and $\mathbf{a}^{\dagger}$, respectively, as

$$
\mathbf{a} \equiv(\mathbf{N}+1)^{1 / 2} \mathbf{E}, \quad \mathbf{a}^{\dagger} \equiv \mathbf{E}^{\dagger}(\mathbf{N}+1)^{1 / 2}
$$

It is clear that $\mathbf{N}=\mathbf{a}^{\dagger} \mathbf{a}$, and

$$
\left[\mathbf{a}^{\dagger}, \mathbf{a}\right]=\sum_{n=0}^{\infty}|n\rangle\langle n|=1_{\mathrm{ob}}
$$

where the $1_{\mathrm{ob}}$ indicates a unit operator in $\mathcal{H}_{o b}$ (notice that it is not a unit operator in $\mathcal{H}$ ). To write the interaction term (3.7) with the operators $\mathbf{E}$ and $\mathbf{E}^{\dagger}$ defined in Eq. (3.11) requires knowledge of the quantum phase problem. Hence, before we continue with the formalism, let us first review this problem in accordance with Lynch 23. 


\section{The Quantum Phase Problem}

Classically, the electric field vector $\vec{E}(\vec{r}, t)$, of an electromagnetic field contained in a cubic cavity of edge L, may be expanded in Fourier components:

$$
\vec{E}(\vec{r}, t) \propto \sum_{\vec{k}}\left(a_{\vec{k}} \mathrm{e}^{i(\vec{k} \cdot \vec{r}-\omega t)}+a_{\vec{k}}^{*} \mathrm{e}^{-i(\vec{k} \cdot \vec{r}-\omega t)}\right)
$$

where we assumed for simplicity a linearly polarized field at a single frequency $\omega$. The sum is taken over all the plane wave modes, where $\vec{k}$ is the wave vector. If we are limited to a single excited mode, say, $\vec{k}=\vec{k}_{0}$, we can write

$$
\vec{E}(\vec{r}, t) \propto\left(a \mathrm{e}^{i\left(\overrightarrow{k_{0}} \cdot \vec{r}-\omega t\right)}+a^{*} \mathrm{e}^{-i\left(\overrightarrow{k_{0}} \cdot \vec{r}-\omega t\right)}\right)
$$

where $a \equiv a_{\overrightarrow{k_{0}}}$. Writing $a=r \mathrm{e}^{i \phi}$ we finally obtain

$$
\vec{E}(\vec{r}, t)=\vec{E}_{0} \cos \left(\overrightarrow{k_{0}} \cdot \vec{r}-\omega t+\phi\right) .
$$

In this way we decompose the classical electric field into amplitude and phase components.

In quantum mechanics $a \rightarrow \mathbf{a}$ and $a^{*} \rightarrow \mathbf{a}^{\dagger}$ where $\mathbf{a}$ and $\mathbf{a}^{\dagger}$ satisfy the canonical commutation relation (3.13). Dirac (1927) was the first to attempt to define a phase operator. He decomposed $\mathbf{a}$ into an amplitude and a phase component

$$
\mathbf{a}=\mathrm{e}^{i \phi} \mathbf{N}^{1 / 2}
$$

where $\mathbf{N}=\mathbf{a}^{\dagger} \mathbf{a}$. The commutator $\left[\mathbf{a}, \mathbf{a}^{\dagger}\right]=1$ leads to the Lerner criterion

$$
\left[\mathrm{e}^{i \phi}, \mathrm{N}\right]=\mathrm{e}^{i \phi} \text {. }
$$

Expanding $\mathrm{e}^{i \phi}$ on both sides of relation 3.18 gives

$$
[\mathbf{N}, \phi]=\mathrm{i}
$$

and thus shows that $\mathbf{N}$ and $\phi$ are canonically conjugate operators. This relation leads to the number - phase uncertainty relation of quantum optics:

$$
\Delta \mathbf{N} \Delta \phi \geq \frac{1}{2}
$$

That is, to create a radiation with a sharp phase, such as a laser beam, we would need to have many photons. Similarly, as we shall see later, a massive BH (analog of many quanta) has a sharp phase.

But Eq. (3.19) leads to serious problems. For example, if we take its matrix elements in the number state basis, we find that

$$
\left(n-n^{\prime}\right)\left\langle n|\phi| n^{\prime}\right\rangle=i \delta_{n, n^{\prime}}
$$

which leads to the impossible equation $0=\mathrm{i}$ for $n=n^{\prime}$. Moreover, Susskind and Glogower 33] have shown that $\mathrm{e}^{i \phi}$ is not unitary, or equivalently, $\phi$ is not Hermitian. 
The recent paper by Bojowald, Kastrup, Schramm and Strobl [28] suggests a solution to these problems. However, as we shall see shortly, despite these problems, if one does not define the phase as an operator (just as time is not an operator in quantum mechanics), the above criticism is not a decisive objection to the phase-number uncertainty relation (3.20). For although we have no time operator, we have time-energy uncertainty relation. The reason why it is difficult to define the phase or the time operator is the fact that the particle number or energy spectrum is non-negative and, therefore, is bounded from below. This situation is drastically different from the one for position and momentum whose eigenvalue spectra are unbounded.

Let us now compare the Dirac definition for the phase operator Eq.(3.17), and Eq. (3.12). One can see that $\mathbf{E}$ and $\mathbf{E}^{\dagger}$ are analogous of $\mathrm{e}^{i \phi}$ and $\mathrm{e}^{-i \phi}$ respectively. Now, using the definition Eq. (3.11) it is easy to show that $\mathbf{E}$ and $\mathbf{E}^{\dagger}$ are "one-sided unitary" or an isometry. That is,

$$
\mathbf{E E}^{\dagger}=1 \text { but } \mathbf{E}^{\dagger} \mathbf{E}=1-|0\rangle\langle 0| .
$$

Despite the fact that the vacuum projection "spoils" the unitarity of $\mathbf{E}$, for states with a very large number occupations (that is, $\langle\mathbf{N}\rangle \gg 1$ ) and hence small vacuum component, we can treat $\mathbf{E}$ as an approximately unitary operator. As we shall see now, the last statement helps us to define the phase of a $\mathrm{BH}$.

\section{The Phase of a Black Hole}

For a $\mathrm{BH}$ not near the Planck mass, $\langle\mathbf{N}\rangle \gg 1$. Thus we can define a phase operator by

$$
\mathrm{e}^{i \phi} \approx \mathbf{E} .
$$

Hence, the interaction term $\mathbf{V}$, which has the form of Eq. (3.7), describes an interaction between the area operator $\mathbf{A}$ and the phase operator $\phi$. As we shall see later, the HR in our model is due to an area-phase interaction.

The definition of the quantum phase of a $\mathrm{BH}$ in Eq. (3.23) raises the question, what is the physical interpretation of the "new" observable $\phi$ ? Furthermore, what are the physical grounds for an area-phase interaction appearing in the Hamiltonian? In order to answer these questions, we first note that the phase operator is the canonical conjugate of the area operator (see Eq. (3.19)). This is reminiscent of work by Carlip and Teitelboim [22].

Carlip and Teitelboim have shown by using the standard (Euclidean) action principle for gravitational field, that the horizon area and the opening angle at the horizon are canonical conjugates. They start with the wave functional $\psi=\psi\left[T,{ }^{(3)} g\right]$ where the separation time at spatial infinity $T$, is an extra argument (in addition to the intrinsic geometry of a spatial section ${ }^{(3)} \mathrm{g}$ ) since space is not closed. The canonical conjugation relation of $T$ and the ADM mass $M$ is expressed in the Schrödinger equation

$$
\frac{\hbar}{i} \frac{\partial \psi}{\partial T}+M \psi=0
$$

which can be obtained from an extended Wheeler-DeWitt equation (that is, the appropriate action is the canonical action $S_{m+g}$ supplemented by $\left.-T M\right)$. In the same manner they 
introduce an extra parameter $\Theta$ ("dimensionless internal time") associated with the horizon by the relation

$$
\frac{\hbar}{i} \frac{\partial \psi}{\partial \Theta}-A \psi=0
$$

where $A$ is the horizon area. They work in a system of polar coordinates $r, \tau$ in $\Re^{2}$ (the spacetime topology is $\Re^{2} \times S^{d-2}$ ) where they set the origin $r=r_{+}$to be the horizon of the BH and the angle $\tau$ to be the Killing time (for more details see Carlip and Teitelboim paper [22]). Next, they show that the parameter $i \Theta$ is " the total proper angle (proper length divided by proper radius) of an arc of very small radius and coordinate angular opening $\tau_{1}-\tau_{2}$ ".

The main result of Carlip and Teitelboim is that the canonical conjugate of the horizon area is the opening angle. The Euclidean continuation of the hyperbolic angle $\Theta$ is bounded and even periodic. This not only favors our simple model, but it gives a motivation for the assumption that the horizon area (in the Euclidean sector) can be represented by a number operator! We are able to define the phase in Eq. (3.23) since we have assumed that the area spectrum is equally spaced. If, for example, the area spacing $a_{n+1}-a_{n}$ approached zero for large quantum number $n$ (as suggested by Ashtekar and others [4]), the quantity canonically conjugate to area would not be a phase operator (its eigenvalues would not be bounded).

In our simple model, the canonical conjugate of the horizon area is also some kind of an angle. Since in our model the phase (angle) is an operator, we shall identify the eigenvalues of $\phi$ with the "opening angles" at the horizon, as discussed by Carlip and Teitelboim. As we shall see below, a further analogy between our approach and theirs can be found in the description of the HR.

Recently, Massar and Parentani [34] have shown that the probability for a BH to emit a particle is given by

$$
P_{M \rightarrow M-\lambda}=N(\lambda, M) \mathrm{e}^{-\Delta A(\lambda, M) / 4}
$$

where $\Delta A$ is the change in the horizon area induced by the emission, and $N$ is some phase space factor. Since they did not neglect the specific heat of the BH, expression (3.26) improves Hawking's result. We shall now point out the origin of this result.

As suggested by Carlip and Teitelboim, Massar and Parentani have supplemented the canonical action with two boundary terms, $-T M$ (boundary at infinity) and $\Theta A / 8 \pi$ (boundary at the horizon). Hence, they face four different actions according to which quantities are fixed in the variational principle. They fix the ADM mass $M$ and the opening angle $\Theta$ in order to describe a physical situation in which the $\mathrm{BH}$ and the surrounding matter exchange energy, but no energy is exchanged with infinity (this is also the process we are studying). It is through the boundary term $\Theta A / 8 \pi$ that they obtain Eq. (3.26).

In our approach, as we shall see later, the HR appears as a result of the interaction (3.7), an interaction between the phase ("opening angle") and the area of the BH. And Massar and Parentani have obtained Eq. (3.26) after introducing the boundary term $\Theta A / 8 \pi$ in the action; this can be considered (in the context of our model) as a 'coupling' between the horizon area and the opening angle. Hence the Massar-Parentani approach is compatible with our model. For this reason we identify the area-phase interaction term in Eq. (3.7) with the boundary term $\Theta A / 8 \pi$. Note that in our approach the interaction term appears in the Hamiltonian, whereas in the Massar-Parentani approach the boundary term appears in 
the action. Furthermore, it is interesting to note that the effect of general relativity (that is, curved spacetime) appears in our simple model as a coupling between the observable $\mathbf{A}$ and its own canonical conjugate $\phi$ !

We now ask what our model has to say about radiation by the $\mathrm{BH}$. In addition to the Hamiltonian we need specify the state of the BH.

\section{THE INITIAL STATE}

One might think that the initial state is just the an area eigenstate $\left|n_{0}\right\rangle$ where $M=$ $m_{0} \sqrt{n_{0}}$ is the initial mass of the BH. But as we shall see in section V1, an area eigenstate describes a static BH (that is, no HR). The question is then, what is the initial state which leads to the semi-classical radiation (HR), and why an area eigenstate is not an appropriate candidate.

The question above is not unique to our problem, but is a general question in quantum mechanics: What are the states that most closely imitate classical behavior? The simplest case where this question appears is that of the simple harmonic oscillator. Hence we shall first review, very shortly, what are the states that imitates the classical harmonic oscillations (see Sakurai).

In the Heisenberg picture the position operator $\mathbf{x}(t)$ of an harmonic oscillator is given by

$$
\mathbf{x}(t)=\mathbf{x}(0) \cos \omega t+\left(\frac{\mathbf{p}(0)}{m \omega}\right) \sin \omega t
$$

where $\omega$ is the angular frequency of the classical oscillator and $\mathbf{p}(0)$ is the momentum operator at $t=0$. The expectation value $\langle n|\mathbf{x}(t)| n\rangle$ vanishes because the operators $\mathbf{x}(0)$ and $\mathbf{p}(0)$ change $n$ by \pm 1 and $|n\rangle$ and $|n+1\rangle$ are orthogonal. This result is different from the classical result no matter how large $n$ may be. In order to obtain the classical result, we have to take the expectation value with respect to a coherent state.

Let us perform the Bogoliubov transformation for coherent states [35]:

$$
\mathbf{a} \rightarrow \alpha(\lambda)=\mathbf{a}-\lambda
$$

where $\lambda$ is a c-number. Then, a coherent state is defined as the vacuum for $\alpha(\lambda)$, and is denoted by $|0(\lambda)\rangle$ :

$$
\alpha(\lambda)|0(\lambda)\rangle=0 \text { or } \mathbf{a}|0(\lambda)\rangle=\lambda|0(\lambda)\rangle \text {. }
$$

We then have

$$
\alpha(\lambda)=\mathbf{U}_{c}(\lambda) \mathbf{a U}_{c}^{-1}(\lambda) \text { with } \quad \mathbf{U}_{c}(\lambda)=\exp \left(i \mathbf{G}_{c}(\lambda)\right)
$$

in which the generator $\mathbf{G}_{c}(\lambda)$ is given by:

$$
\mathbf{G}_{c}(\lambda)=i\left(\lambda^{*} \mathbf{a}-\mathbf{a}^{\dagger} \lambda\right) .
$$

We note parenthetically that if we allow $\lambda$ to be a function of $\mathbf{A}$, we shall be able to express the interaction term in Eq. (3.7) as the generator of BH coherent states. That is, 


$$
\mathbf{V}=\mathbf{G}_{c}(\lambda(\mathbf{A})) \quad \text { with } \quad \lambda(\mathbf{A})=i f^{*}(\mathbf{A})(\mathbf{N}+1)^{-1 / 2} .
$$

This remarkable result implies that the interaction term given in Eq. (3.7) induces the Bogoliubov transformation (4.2); we shall see that it defines the BH states which exhibit HR.

Let us now list two properties of a coherent state which will be relevant to our problem. Firstly, a coherent state may be expressed as a superposition of the number $(\mathbf{N})$ eigenstates

$$
|0(\lambda)\rangle=\mathbf{U}_{c}(\lambda)|0\rangle=\sum_{n=0}^{\infty} q(n)|n\rangle
$$

where the distribution of $|q(n)|^{2}$ with respect to $n$ is of the Poisson type about some mean value $\bar{n}=|\lambda|^{2}$ :

$$
|q(n)|^{2}=\left(\frac{\bar{n}^{n}}{n !}\right) \exp (-\bar{n})
$$

Secondly, a coherent state satisfies the minimum uncertainty product relation.

By analogy with a simple harmonic oscillator, we shall ask how can we construct a superposition of the $\mathrm{BH}$ area eigenstates that most closely imitates the semi-classical result (HR)? In comparison with the harmonic oscillator, would it be some kind of a coherent state? We shall call the state that does the desired job the coherent-termal statef and denote it by $\left|C T ; n_{0}\right\rangle$ where $n_{0} \equiv\left\langle C T ; n_{0}|\mathbf{N}| C T ; n_{0}\right\rangle$. Hence, in the limit of a massive $\mathrm{BH}$ we demand

$$
\lim _{n_{0} \gg 1}\left\langle C T ; n_{0}|\mathbf{M}(t)| C T ; n_{0}\right\rangle=\langle M(t)\rangle
$$

where $\langle M(t)\rangle$ is the result obtained in the semi-classical approach (see section $\mathrm{D}$ ).

An observer at infinity is limited by the energy-time (area-phase) uncertainty principle when he tries to determine the mass (area) of a $\mathrm{BH}$ formed by a gravitational collapse. If the $\mathrm{BH}$ is an eternal one, in which one can determine exactly its mass, there will be no HR because the initial state will be a mass eigenstate (see section $\nabla \mathbb{1}$ ). By contrast, if the $\mathrm{BH}$ has been formed by gravitational collapse, there is always radiation in the region between the $\mathrm{BH}$ and the observer. Even if hypothetically at some initial time, say $t_{0}$, there is no radiation, still the measurement takes time, say $\Delta t$, in which a small amount of radiation is produced according to the mass-time uncertainty principle. Hence, in order to be compatible with the HR, the initial state must satisfy $\left\langle(\Delta \mathbf{M})^{2}\right\rangle \neq 0$, or equivalently, the HR is due to the uncertainty (of an observer at infinity) about the area (mass) of the $\mathrm{BH}$.

The coherent-thermal state (CT-state) may be expressed as follows:

$$
\left|C T ; n_{0}\right\rangle=\sum_{n=0}^{\infty} P_{n}^{1 / 2} \exp (-i \nu(n))|n\rangle
$$

where $P_{n}$ is the distribution with respect to $n$ and $\exp (-i \nu(n))$ is some phase coefficient where $\nu(n)$ is a real function of $n$. We can set $\exp (-i \nu(n))=1$ by defining $|n\rangle_{\text {new }} \equiv$

\footnotetext{
${ }^{2}$ The termal feature of this state has been actually erased after performing the projection operation of the horizon.
} 
$\exp (-i \nu(n))|n\rangle$ and doing all the previous analysis with respect to these new states. The new Susskind-Glogower's operators, $\mathbf{E}$ and $\mathbf{E}^{\dagger}$, are different from the old ones, but the form of the Hamiltonian and the interaction term given in Eq. (3.7) are unchanged since $f(\mathbf{A})$ can absorb the changes. Hence we conclude that the CT-state may be written as:

$$
|C T ; \bar{n}\rangle=\sum_{n=0}^{\infty} P_{n}^{1 / 2}|n\rangle .
$$

We are interested in the semi-classical limit $n_{0} \rightarrow \infty$. In this limit $\sigma_{M} /\langle\mathbf{M}\rangle \rightarrow 0$ where $\sigma_{M}^{2} \equiv\left\langle M^{2}\right\rangle-\langle M\rangle^{2}$. Hence, in the limit of large $n_{0}$ value, the distribution $P_{n}$ is of the Gaussian type about the mean value $n_{0}$ [36]. That is,

$$
P_{n} \approx\left(\frac{\alpha}{4 \pi n_{0}}\right)^{\frac{1}{2}} \exp \left(-\frac{\alpha}{4 n_{0}}\left(n-n_{0}\right)^{2}\right)
$$

where we have left the variance, $\sigma_{A}^{2}=2 n_{0} / \alpha$, to be determined later. But a distribution of the Poisson type (see Eq. (4.8)) becomes, in the limit $n_{0} \rightarrow \infty$, Gaussian with a variance $\sigma_{A}^{2}=n_{0}$. Thus, for a coherent state $\alpha=2$. However, to be more general we shall not yet set $\alpha=2$.

Let us now compare the semi-classical results and our quantum mechanical approach when the averages are taken with respect to the CT-state

$$
\left|C T ; n_{0}\right\rangle=\left(\frac{\alpha}{4 \pi n_{0}}\right)^{\frac{1}{4}} \sum_{n=0}^{\infty} \exp \left(-\frac{\alpha}{8 n_{0}}\left(n-n_{0}\right)^{2}\right)|n\rangle .
$$

\section{THE ROLE OF HAWKING RADIATION}

We start by emphasizing the similarity of the following two roads to the HR: (1)If one attributes to a $\mathrm{BH}$ an entropy $S_{B H}=A / 4 \mathcal{L}_{P}^{2}$ (Bekenstein [12]), it leads to a temperature $T_{B H}=\hbar / 8 \pi M$ (Hawking [17]); hence the $\mathrm{BH}$ radiates and decreases its horizon area $\left(A=16 \pi M^{2}\right)$. Due to the thermal character of the radiation, one can calculate the rate of change of the BH mass using Stefan-Boltzmann law (see Eq. (5.1)).

(2)BH entropy, together with the assumption that the horizon area is represented by the number operator $\mathbf{A}=4 \ln k \mathcal{L}_{P}^{2} \mathbf{N}$, implies a huge degeneracy with respect to $\mathbf{N}, k^{n}$. This degeneracy motivated us to introduce into the Hamiltonian an interaction term of the form of Eq. (3.6). A BH with such high degeneracy acts in order to reduce its internal degrees of freedom. Hence the area of the $\mathrm{BH}$ is not conserved, i.e. $[\mathbf{H}, \mathbf{A}] \neq 0$. Later on we shall see that it is indeed possible to choose the interaction term such that the area of the $\mathrm{BH}$ decreases in time.

The purpose of this section is to compare these two roads. Such a comparison examines the validity of our assumptions that lead to the form of the Hamiltonian given in the previous section. Moreover, we shall be able to derive the form of the function $f(\mathbf{A})$ in Eq. (3.7) for large $\langle\mathbf{A}\rangle$ values. We shall point out that these two roads should match only in the limit of large $\langle\mathbf{A}\rangle$ values (the semi-classical limit). For much smaller values of $\langle\mathbf{A}\rangle$ (near the Planck scale), our approach predicts new results. 
Due to Hawking radiance and its thermal character, it is possible to estimate the rate of change of the mass $\langle M\rangle$ of the BH. Using the Stefan-Boltzmann law for a surface area $16 \pi\langle M\rangle^{2}$ at temperature $T_{B H}=(\hbar / 8 \pi)\langle M\rangle^{-1}$, we find (see Bekenstein and Mukhanov [7] and Page 25])

$$
\frac{d\langle M\rangle}{d t}=-\frac{\gamma \hbar}{15360 \pi\langle M\rangle^{2}} \equiv-\frac{\varepsilon}{\langle M\rangle^{2}}
$$

where $\gamma$ lumps uncertainties about the species of particles emitted. We shall assume that the mass of the $\mathrm{BH}$ at time $t=0$ is equal to the total energy of the system, that is, $\langle M(0)\rangle$ is the ADM mass. Now, solving Eq. (5.1) we find

$$
\langle M(t)\rangle=\langle M(0)\rangle\left(1-\frac{3 \varepsilon t}{\langle M(0)\rangle^{3}}\right)^{1 / 3}=\langle M(0)\rangle-\frac{\varepsilon}{\langle M(0)\rangle^{2}} t-\frac{\varepsilon^{2}}{\langle M(0)\rangle^{5}} t^{2}-\cdots
$$

where $\varepsilon$ is defined in Eq. (5.1) and the mass of the BH is assumed to be very large compared to $(\varepsilon t)^{3}$.

In our model, the mass of the $\mathrm{BH}$ is represented by the mass operator $\mathbf{M} \equiv \mathbf{M}_{S} \equiv \mathbf{M}_{H}(0)$ where the subscripts $S$ and $H$ stand for the Schrödinger and Heisenberg pictures, respectively. The evolution in time of the mass operator in the Heisenberg picture is determined by

$$
\begin{aligned}
\mathbf{M}_{H}(t) & =\exp \left(\frac{i}{\hbar} \mathbf{H} t\right) \mathbf{M}(0) \exp \left(-\frac{i}{\hbar} \mathbf{H} t\right)=\mathbf{M}(0)+\frac{i}{\hbar}[\mathbf{H}, \mathbf{M}(0)] t \\
& +\frac{1}{2 !}\left(\frac{i}{\hbar}\right)^{2}[\mathbf{H},[\mathbf{H}, \mathbf{M}(0)]] t^{2}+\cdots
\end{aligned}
$$

where the Hamiltonian is given by Eq. (3.3) and Eq. (3.7). In order to compare Eq. (5.3) with the semi-classical result (5.2), we have to take the average of both sides of Eq. (5.3). We shall first compare between the coefficients that are proportional to $t$ in both Eq. (5.2) and (the mean value of) Eq. (5.3).

\section{A. The First Order Corrections}

The Hamiltonian (3.3) may be written as

$$
\mathbf{H}=\mathbf{M}(0)+\sum_{n=0}^{\infty}\left(f(n)|n\rangle\left\langle n+1\left|+f^{*}(n)\right| n+1\right\rangle\langle n|\right)
$$

where we used Eq. (3.7) for V. Hence it clear that

$$
\begin{aligned}
& {[\mathbf{H}, \mathbf{M}(0)]=[\mathbf{V}, \mathbf{M}(0)]=} \\
& m_{0} \sum_{n=0}^{\infty}\left((\sqrt{n+1}-\sqrt{n})\left(f(n)|n\rangle\left\langle n+1\left|-f^{*}(n)\right| n+1\right\rangle\langle n|\right)\right) .
\end{aligned}
$$

Note that if we take the average with respect to an area eigenstate $|n\rangle$, we would find that

$$
\langle n|[\mathbf{H}, \mathbf{M}(0)]| n\rangle=0,
$$


and it would be impossible to compare with Eq. (5.2).

Now, taking the average with respect to the CT-state (4.13) we find

$$
\begin{aligned}
& \langle[\mathbf{H}, \mathbf{M}(0)]\rangle_{C T ; n_{0}}=2 m_{0} \sum_{n=0}^{\infty} f(n)(\sqrt{n+1}-\sqrt{n})\langle\mid n\rangle\langle n+1 \mid\rangle_{C T ; n_{0}}= \\
& 2 m_{0}\left(\frac{\alpha}{4 \pi n_{0}}\right)^{\frac{1}{2}} \sum_{n=0}^{\infty} f(n)(\sqrt{n+1}-\sqrt{n}) \mathrm{e}^{\left(-\frac{\alpha}{8 n_{0}}\left(n+1-n_{0}\right)^{2}-\frac{\alpha}{8 n_{0}}\left(n-n_{0}\right)^{2}\right)} \\
& =2 m_{0} \mathrm{e}^{-\frac{\alpha}{16 n_{0}}}\left(\frac{\alpha}{4 \pi n_{0}}\right)^{\frac{1}{2}} \sum_{n=0}^{\infty} f(n)(\sqrt{n+1}-\sqrt{n}) \mathrm{e}^{\left(-\frac{\alpha}{4 n_{0}}\left(n-n_{0}+\frac{1}{2}\right)^{2}\right)} \\
& \stackrel{n_{0} \gg 1}{\approx} 2 m_{0} f\left(n_{0}\right)\left(\sqrt{n_{0}+1}-\sqrt{n_{0}}\right) \mathrm{e}^{\left(-\frac{\alpha}{16 n_{0}}\right)}
\end{aligned}
$$

where we have assumed that $f(n)$ is purely imaginary since we want that $\langle\mathbf{H}\rangle_{C T ; n_{0}}=$ $\langle\mathbf{M}(0)\rangle_{C T ; n_{0}}$ (the mass of the $\mathrm{BH}$ at time $t=0$ is equal to the total energy of the system). Now, comparing $\mathrm{i} \hbar^{-1}\langle[\mathbf{H}, \mathbf{M}(0)]\rangle_{C T ; n_{0}}$ with the coefficient of $t$ in Eq. (5.2) we find

$$
f\left(n_{0}\right) \stackrel{n_{0} \gg 1}{=} \frac{i \hbar \varepsilon}{m_{0}^{2}\langle M(0)\rangle} \mathrm{e}^{\left(\frac{\alpha}{16 n_{0}}\right)}
$$

where we have taken $\sqrt{n_{0}+1}-\sqrt{n_{0}} \approx 1 /\left(2 \sqrt{n_{0}}\right)$ since $n_{0}$ is very large. Because $\langle M(0)\rangle=$ $m_{0} \sqrt{n_{0}}$ we conclude

$$
f(n) \stackrel{n \geqq 1}{\frac{i \hbar \varepsilon}{m_{0}^{3}}} \mathrm{e}^{\left(\frac{\alpha}{16 n_{0}}\right)} \frac{1}{\sqrt{n}} .
$$

Note that the $n_{0}$ in the exponent was not replaced by $n$ because this exponent originally came from the initial state (see Eq. (5.7) before taking the limit of large $n_{0}$ ). This equation holds only for $n \gg 1$ and there are corrections in the limit of small $n$. Note that this result is in harmony with our previous estimate of $f(\mathbf{A})$ as proportional to $1 / \sqrt{\mathbf{A}}$ (see the arguments below Eq. (3.9)).

It is clear that the function $f(n)$ should not be dependent on the CT-state characterized by the number $n_{0}$ (otherwise, the Hamiltonian would be dependent on the states we take average with). Hence, the factor $\exp \left(\frac{\alpha}{16 n_{0}}\right)$ should be independent of $n_{0}$. There are two ways to achieve this. One is to assume that $\alpha$ is proportional to $n_{0}$ such that the ratio $\alpha / n_{0}$ is independent of $n_{0}$. The other way is to assume that $\alpha$ is of the order of unity, and since our calculations are valid up to corrections of $\mathrm{O}\left(1 / n_{0}\right)$, we can replace $\exp \left(\frac{\alpha}{16 n_{0}}\right)$ by one. By contrast, it would be incorrect to replace $\exp \left(\frac{\alpha}{16 n_{0}}\right)$ by unity if, for example, $\alpha \sim \sqrt{n_{0}}$. The reason is that we cannot neglect corrections of $\mathrm{O}\left(1 / \sqrt{n_{0}}\right)$ and thus the function $f(n)$ will be dependent on $n_{0}$ by a small correction $\left(\sim 1 / \sqrt{n_{0}}\right)$ which cannot be neglected. Since the Hamiltonian should not depend on $n_{0}$ (even not as a small correction), we conclude that either $\alpha$ is of the order of unity, or it is of the order of $n_{0}$. The question now is which one of the two possibilities for $\alpha$ is the correct one.

One may ask if the area-phase uncertainty relation (3.20) can supply the required information about $\alpha$. As we have mentioned earlier, a coherent state (e.g. the initial CT- state with $\alpha=2$ ) satisfies the minimum uncertainty product relation. In Appendix C, we show 
that both of the choices for $\alpha$ lead to the minimum uncertainty product relation (3.20). Thus we are left with an unknown parameter.

As we shall see shortly, Eq. (5.9) leads to a second order correction of the form $\sim t^{2} /\langle M(0)\rangle^{5}$ for the evaporation of the black hole [see Eq. (5.2)]. This result is, surprisingly, consistent with the semi-classical result Eq. (5.2). Hence, we shall compare also the coefficients of second order in both approaches, and deduce restrictions on $\alpha$.

\section{B. The Second Order Corrections}

In order to calculate the second order of $\langle\mathbf{M}(t)\rangle$ with respect to the time $t$, we use Eq. (5.3) and calculate the average of

$$
[\mathbf{H},[\mathbf{H}, \mathbf{M}(0)]]=[\mathbf{M}(0),[\mathbf{V}, \mathbf{M}(0)]]+[\mathbf{V},[\mathbf{V}, \mathbf{M}(0)]]
$$

with respect to the CT-state. Due to the identity $\langle\mid n\rangle\langle n+1 \mid\rangle=\langle\mid n+1\rangle\langle n \mid\rangle$, the first term in Eq. (5.10) averages out to zero. To calculate the average of the second term in Eq. (5.10), we substitute Eq. (5.8) in the expression for V. Thus, we find

$$
\begin{aligned}
\langle[\mathbf{V},[\mathbf{V}, \mathbf{M}(0)]]\rangle_{C T ; n_{0}}= & \frac{\hbar^{2} \varepsilon^{2}}{m_{0}^{5}} \mathrm{e}^{\left(\frac{\alpha}{8 n_{0}}\right)} \sum_{n>1}^{\infty}\left\{\left(\frac{1}{n^{3 / 2}}-\frac{1}{(n+1)^{3 / 2}}\right)\langle\mid n\rangle\langle n \mid\rangle_{C T ; n_{0}}\right. \\
& \left.+\left(\frac{1}{n \sqrt{n+1}}-\frac{1}{(n+1) \sqrt{n}}\right)\langle\mid n+2\rangle\langle n \mid\rangle_{C T ; n_{0}}\right\} \\
= & \frac{\hbar^{2} \varepsilon^{2}}{2\langle M(0)\rangle^{5}} \mathrm{e}^{\left(\frac{\alpha}{8 n_{0}}\right)}\left(3+\sum_{n=0}^{\infty}\langle\mid n+2\rangle\langle n \mid\rangle_{C T ; n_{0}}\right) \\
= & \frac{\hbar^{2} \varepsilon^{2}}{2\langle M(0)\rangle^{5}}\left(3 \mathrm{e}^{\left(\frac{\alpha}{8 n_{0}}\right)}+\mathrm{e}^{\left(-\frac{\alpha}{8 n_{0}}\right)}\right)
\end{aligned}
$$

where we have used the fact that the contribution of small $n$ 's (compared to $n_{0}$ ) may be neglected under the average. Finally, comparing Eq. (5.11) with the semi-classical result (5.2) we find

$$
3 \mathrm{e}^{\left(\frac{\alpha}{8 n_{0}}\right)}+\mathrm{e}^{\left(-\frac{\alpha}{8 n_{0}}\right)}=4
$$

Note that Eq. (5.12) has two solutions. One is $\exp \left(\alpha / 8 n_{0}\right)=1$. This solution is plausible, up to a very good approximation, if $\alpha$ is of order of unity since then $\exp \left(\alpha / 8 n_{0}\right)=1+\mathrm{O}\left(1 / n_{0}\right)$. A coherent state, that is $\alpha=2$, also satisfies Eq. (5.12). This remarkable result shows that our model for the Stefan-Boltzmann law agrees with the semi-classical result up to the second order in time. We have used the first order correction to obtain $f(n)$, and this allowed us to reproduce exactly the second order correction (including the non-dimensional numerical coefficient).

The other solution of Eq. (5.12) is $\exp \left(\alpha / 8 n_{0}\right)=\frac{1}{3}$ which is impossible for $\alpha>0$. Hence, it seems at first sight that a solution for $\alpha$ of order $n_{0}$ is impossible.

In the last argument we have assumed that the interaction term in Eq. (3.7) is exact. If one insists that the initial CT-state, which imitates the semi-classical result, is such that $\alpha \sim n_{0}$ (and not of the order of unity), we must then add a correction to the Hamiltonian. 
In appendix B we have estimated the second order correction (if there is one) to the Hamiltonian, and we have reproduced Eq. (5.12) with a correction coming from the extra term in the Hamiltonian. We shall emphasize here that the second order correction contributes Eq. (5.12) because we have calculated $\langle\mathbf{M}(t)\rangle$ up to a second order in time.

\section{STATIC BLACK HOLES}

At first glance it seems impossible to describe a static $\mathrm{BH}$ in our model since $[\mathbf{H}, \mathbf{A}] \neq 0$. But, as we have seen in section $\mathrm{VA}$, the first order correction to the rate of change of the mass of the $\mathrm{BH}$ is zero when the initial state is taken to be an area eigenstate. Thus, area eigenstates seem to be good candidates for describing eternal BHs. Note that these states satisfy $\left\langle(\Delta \mathbf{M})^{2}\right\rangle=0$ which is compatible with eternal BHs since no gravitational collapse is involved (see section [V]). As we shall see in this section, for a $\mathrm{BH}$ (not a primordial one) which is described by an area eigenstate, we have to wait a long time (much greater than the age of the universe) in order to observe any change in its horizon area, and even then it will only be a small fluctuation about the mean area value.

The idea that area eigenstates describe static BHs has interesting implications. For example, if one had a 'device' able to measure the area of the $\mathrm{BH}$ (that is, the $\mathrm{BH}$ state collapse into an area eigenstate), it would, by measuring it, stop the time evolution of the $\mathrm{BH}$ ! This means that our knowledge about the area of the BH plays a central role, and affects its mass evaporation.

We start with the Hamiltonian given in Eq. (5.4) where $f(n)$ is given by Eq. (5.9) for large $n$. As we have done in the previous sections, since $f(n)$ approaches zero for large $n$ values, we shall treat the interaction term in a perturbative manner. This time we shall work in the interaction picture.

The time dependent "potential" in the interaction picture is given by

$$
\mathbf{V}_{I}(t)=\exp \left(\frac{i}{\hbar} \mathbf{M}(0) t\right) \mathbf{V} \exp \left(-\frac{i}{\hbar} \mathbf{M}(0) t\right)
$$

where $\mathbf{V}$ is the interaction term given in Eq. (5.4). The time evolution operator (in the interaction picture) is then expressed as

$$
\begin{aligned}
\mathbf{U}_{I}(t) & =\mathcal{T} \exp \left(-i \hbar^{-1} \int_{0}^{t} \mathbf{V}_{I}\left(t^{\prime}\right) d t^{\prime}\right) \\
& =1-\frac{i}{\hbar} \int_{0}^{t} \mathbf{V}_{I}\left(t^{\prime}\right) d t^{\prime}+\left(-\frac{i}{\hbar}\right)^{2} \int_{0}^{t} d t^{\prime} \int_{0}^{t^{\prime}} d t^{\prime \prime} \mathbf{V}_{I}\left(t^{\prime}\right) \mathbf{V}_{I}\left(t^{\prime \prime}\right)+\cdots
\end{aligned}
$$

where $\mathcal{T}$ is the time ordering operator. We proceed to evaluate the average of the area operator when the initial state is the area eigenstate $\left|n_{0}\right\rangle$. We shall calculate the average up to the first non vanishing order. That is,

$$
\begin{aligned}
\langle\mathbf{A}\rangle_{t} & =\left\langle n_{0}\left|\mathbf{U}_{I}^{\dagger}(t) \mathbf{A} \mathbf{U}_{I}(t)\right| n_{0}\right\rangle=n_{0} a_{0} \\
& +\frac{1}{\hbar^{2}}\left\langle n_{0}\left|\int_{0}^{t} \mathbf{V}_{I}\left(t^{\prime}\right) d t^{\prime} \mathbf{A} \int_{0}^{t} d t^{\prime \prime} \mathbf{V}_{I}\left(t^{\prime \prime}\right)\right| n_{0}\right\rangle \\
& -\frac{n_{0} a_{0}}{\hbar^{2}}\left\langle n_{0}\left|\int_{0}^{t} d t^{\prime} \int_{0}^{t^{\prime}} d t^{\prime \prime} \mathbf{V}_{I}\left(t^{\prime}\right) \mathbf{V}_{I}\left(t^{\prime \prime}\right)\right| n_{0}\right\rangle+\mathrm{O}\left(\mathbf{V}_{I}^{4}\right)
\end{aligned}
$$


where we have used Eq. (6.2) (note that the area operator in the interaction picture is the same as in Schrödinger picture).

Now, using Eq. (6.1) and Eq. (5.4) to express $\mathbf{V}_{I}(t)$, we find after some algebraic manipulations that Eq. (6.3) can be written as

$$
\begin{aligned}
\langle\mathbf{A}\rangle_{t} & =n_{0} a_{0} \\
& -\frac{4 a_{0}}{\hbar^{2}}\left(\frac{\left|f\left(n_{0}-1\right)\right|^{2}}{\omega_{n_{0}, n_{0}-1}^{2}} \sin ^{2}\left(\frac{1}{2} \omega_{n_{0}, n_{0}-1} t\right)-\frac{\left|f\left(n_{0}\right)\right|^{2}}{\omega_{n_{0}, n_{0}+1}^{2}} \sin ^{2}\left(\frac{1}{2} \omega_{n_{0}, n_{0}+1} t\right)\right)
\end{aligned}
$$

where

$$
\omega_{n_{0}, n_{0} \pm 1} \equiv \frac{E_{n_{0}}-E_{n_{0} \pm 1}}{\hbar} \equiv \frac{m_{0} \sqrt{n_{0}}-m_{0} \sqrt{n_{0} \pm 1}}{\hbar}
$$

Now, because $f\left(n_{0}\right)$, and $\omega_{n_{0}, n_{0} \pm 1}$ are of $\mathrm{O}\left(1 / \sqrt{n_{0}}\right)$ for $n_{0} \gg 1$, we find that $\mid f\left(n_{0}-\right.$ 1) $\left.\right|^{2} / \omega_{n_{0}, n_{0}-1}^{2}$ is equal to $\left|f\left(n_{0}\right)\right|^{2} / \omega_{n_{0}, n_{0}+1}^{2}$ up to a very small correction of the order of $1 / n_{0}$. Thus, after using Eq. (5.9) for $f(n)$ we find

$$
\frac{\left|f\left(n_{0}-1\right)\right|^{2}}{\omega_{n_{0}, n_{0}-1}^{2}}=\frac{\left|f\left(n_{0}\right)\right|^{2}}{\omega_{n_{0}, n_{0}+1}^{2}}=\frac{4 \hbar^{4} \varepsilon^{2}}{m_{0}^{8}} \exp \left(\frac{\alpha}{8 n_{0}}\right)
$$

for $n_{0} \gg$ 1. Substituting Eq. (6.6) in Eq. (6.4) we finally obtain

$$
\begin{aligned}
\langle\mathbf{A}\rangle_{t} & =a_{0} n_{0}-a_{0} \xi^{2}\left(\sin ^{2}\left(\frac{1}{2} \omega_{n_{0}, n_{0}-1} t\right)-\sin ^{2}\left(\frac{1}{2} \omega_{n_{0}, n_{0}+1} t\right)\right) \\
& =a_{0} n_{0}-a_{0} \xi^{2} \sin \left(\omega^{(1)} t\right) \sin \left(\omega^{(2)} t\right)
\end{aligned}
$$

where (with $G$ and $c$ displayed)

$$
\omega^{(1)}=\frac{\ln k}{8 \pi M}\left(\frac{c^{3}}{G}\right), \quad \omega^{(2)}=\frac{(\ln k)^{2} \hbar}{128 \pi^{2} M^{3}}\left(\frac{c^{4}}{G^{2}}\right) \quad \text { and } \xi=\frac{\pi \gamma \exp \left(\frac{\alpha}{8 n_{0}}\right)}{240(\ln k)^{2}} .
$$

Note that $\xi$ is a dimensionless coefficient, whereas $\gamma$ was first introduced in Eq. (5.1).

The frequency $\omega^{(1)}$ does not contain $\hbar$; hence we call it the classical frequency of the BH. The second frequency, $\omega^{(2)}$, is the quantum correction to $\omega^{(1)}$, and even for a microscopic BH with $\langle\mathbf{M}(0)\rangle=10^{12} \mathrm{Kg}, \omega^{(2)} \approx 10^{-19} \sec ^{-1}$ (where we assumed $\ln k \sim 1$ ). Thus $\sin \left(\omega^{(2)} t\right) \approx 0$ for $t$ not much larger that the age of the universe. We conclude that a BH (not a primordial one) which is described initially by a pure state $\left|n_{0}\right\rangle$ will stay in this pure state since $\langle\mathbf{A}\rangle_{t}=$ $a_{0} n$ over the lifetime of the universe.

The frequency $\omega^{(2)}$ becomes significant when $1 / \omega^{(2)}$ is smaller then the age of the universe. That is, BHs with a mass smaller than $10^{11} \mathrm{Kg}$. For these BHs it is impossible to neglect $\omega^{(2)}$. But still, if we assume that $\xi$ (or $\gamma$ ) is not extremely large, Eq. (6.7) describes a basically static $\mathrm{BH}$ with small time dependent fluctuations around its mean area value. 


\section{SUMMARY AND CONCLUSIONS}

We have described in this paper a fully quantum mechanical model for a Schwarzschild $\mathrm{BH}$. Starting from the assumption that the horizon area is quantized with equally spaced spectrum, we have obtained for the black hole observables an algebra of angular momentum (the hyperspin of a Schwarzschild $\mathrm{BH}$ ) with the restriction that the hyperspin can only have the values $s_{n}=\left(k^{n}-1\right) / 2$. This serves as motivation for the introduction in the Hamiltonian of an interaction term. Surprisingly, this term leads to the correct properties of the HR.

We have introduced the phase of a $\mathrm{BH}$ as the canonical conjugate to the area operator, and identified it with the "opening angle" of Carlip and Teitelboim [22]. Then the "interaction" term in the Hamiltonian has been interpreted as a coupling between the horizon area and the phase of the $\mathrm{BH}$. This coupling we associated with the boundary term $\Theta A / 8 \pi$ in the action of a $\mathrm{BH}$ spacetime, which according to Massar and Parentani [34] corresponds to the HR.

To discuss the HR it was required to choose an appropriate initial state which most closely imitates the semi-classical result (HR). We have chosen the initial state to be Gaussian distributed with area about some mean value $n_{0}$. Hence, the variance $\sigma_{A}$ of this state was left undetermined. However, after the comparison with the HR, it has been shown that either $\sigma_{A} /\langle\mathbf{A}\rangle \sim \mathcal{M}_{P} / M$, or $\sigma_{A} /\langle\mathbf{A}\rangle \sim\left(\mathcal{M}_{P} / M\right)^{2}$.

For the first possibility, the Hamiltonian which describes the system is exactly of the form of Eq. (5.4). On the other hand, if $\sigma_{A} /\langle\mathbf{A}\rangle \sim\left(\mathcal{M}_{P} / M\right)^{2}$, then the Hamiltonian given by Eq. (5.4) is just part (in a perturbation theory) of the full Hamiltonian of the BH (see Appendix B).

We have seen in the previous section that an area eigenstate describes a static BH. Hence, in our model the $\mathrm{HR}$ is due to the dispersion in the area eigenstates. It was shown long ago by Bekenstein [36] that the fluctuations in the mass of the $\mathrm{BH}$, and thus also in its area, increases with time. Also Wu and Ford [37] have shown recently that the variance of the mass grows linearly in time. It is then clear that as long as the BH looses mass, the

observers loose their knowledge about the area of the $\mathrm{BH}$. Perhaps this is the way that a $\mathrm{BH}$ avoids a singularity at the culmination of its evaporation.

\section{ACKNOWLEDGMENTS}

I would like to thank Prof. J. Bekenstein for his guidance and support during the course of this work. They proved invaluable to me. It is also a pleasure to thank A. E. Mayo, S. Hod and L. Sriramkumar for helpful discussions. This research was supported by a grant from the Israel Science Foundation, established by the Israel National Academy of Sciences.

\section{APPENDIX A: SETTING $U(\mathbf{N})=1$}

In order to prove that $u(\mathbf{N})=1$ in Eq. (2.6), we first define three operators in the same manner as in Eq. (2.14), that is

$$
\mathbf{J}_{\mathbf{1}} \equiv \frac{1}{2}\left(l(\mathbf{N}) \mathbf{g}+\mathbf{g}^{\dagger} l^{*}(\mathbf{N})\right)
$$




$$
\begin{aligned}
\mathbf{J}_{\mathbf{2}} & \equiv \frac{i}{2}\left(l(\mathbf{N}) \mathbf{g}-\mathbf{g}^{\dagger} l^{*}(\mathbf{N})\right) \\
\mathbf{J}_{\mathbf{3}} & \equiv|l(\mathbf{N})|^{2}\left(\mathbf{G}-\frac{1}{2} k^{\mathbf{N}}-\frac{1}{2}\right)
\end{aligned}
$$

where $u(\mathbf{N}) \equiv|l(\mathbf{N})|^{2}$ since $u(\mathbf{N})$ must have real positive eigenvalues (see Eq. (2.6)). These three operators satisfy the angular momentum commutation rules and thus the eigenvalues of $\mathbf{J}_{\mathbf{3}}$ and $\mathbf{J}^{2}$ are $m_{3}=-j,-j+1, \ldots, j$ and $j(j+1)$, respectively, where $j$ is an integer or half integer.

Using Eq. (A1) and Eq. (2.6) one can obtain

$$
4 \mathbf{J}^{2}=|l(\mathbf{N})|^{2}\left(k^{2 \mathbf{N}}-1-\left(1-|l(\mathbf{N})|^{2}\right)\left(2 \mathbf{G}-k^{\mathbf{N}}-1\right)^{2}\right) .
$$

Hence it is clear that the eigenstates $|n, m\rangle$ of $\mathbf{N}$ and $\mathbf{G}$ are also eigenstates of $\mathbf{J}_{3}$ and $\mathbf{J}^{2}$. Thus we shall write $m_{3}$ and $j$ in terms of $n$ and $m$ as follows:

$$
\begin{aligned}
2 m_{3} & =|l(n)|^{2}\left(2 m-k^{n}-1\right) \\
4 j(j+1) & =|l(n)|^{2}\left(k^{2 n}-1-\left(1-|l(n)|^{2}\right)\left(2 m-k^{n}-1\right)^{2}\right) .
\end{aligned}
$$

From Eq. (A3) it follows that $|l(n)|^{2}$ is an integer since $2 m_{3}$ is an integer. Thus, $|l(n)|^{2} \geq 1$.

Setting for example $m=1$ in Eq. (A3) and Eq. (A4) we find that

$$
\begin{aligned}
2 m_{3} & =|l(n)|^{2}\left(1-k^{n}\right) \\
4 j(j+1) & =|l(n)|^{2}\left(k^{n}-1\right)\left(k^{n}+2-|l(n)|^{2}\right) .
\end{aligned}
$$

Now, the eigenvalues of an angular momentum satisfy the condition $2 j \geq\left|2 m_{3}\right|$ which leads to $4 j(j+1) \geq\left|2 m_{3}\right|\left(\left|2 m_{3}\right|+2\right)$. Hence, using both Eq. (A5) and Eq. (A6) we finally obtain

$$
|l(n)|^{2}\left(k^{n}-1\right)\left(k^{n}+2-|l(n)|^{2}\right) \geq|l(n)|^{2}\left(k^{n}-1\right)\left(k^{n}+1\right)
$$

which is satisfied only if $|l(n)|^{2} \leq 1$. Thus we have proved that $u(n) \equiv|l(n)|^{2}=1$.

\section{APPENDIX B: A SECOND ORDER CORRECTION TO THE HAMILTONIAN}

The second order correction is related to transitions $n \rightarrow n \pm 2$ (see section [IIB), and hence may be written as:

$$
\mathbf{H}^{(2)}=\sum_{n=0}^{\infty}\left(r(n)|n+2\rangle\left\langle n\left|+r^{*}(n)\right| n\right\rangle\langle n+2|\right)
$$

where $r(n)$ is a complex function of $n$. Now, since to zeroth order $\mathbf{H}^{(0)} \equiv \mathbf{M} \sim \sqrt{\mathbf{A}}$, and the first order correction $\mathbf{H}^{(1)} \equiv \mathbf{V} \sim 1 / \sqrt{\mathbf{A}}$ for large $\langle\mathbf{A}\rangle$ values, we conclude that for large $\langle\mathbf{A}\rangle$ values the second order correction is proportional to $1 / \mathbf{A}^{3 / 2}$, i.e. each term of a higher order in the perturbation theory is smaller by one extra power of $\mathbf{A}$. Thus, the function $r(n)$ in Eq. (B1) can be written for large $n$ as 


$$
r(n)=\zeta \frac{(\hbar \varepsilon)^{2}}{m_{0}^{5}} \mathrm{e}^{\left(\frac{\alpha}{8 n_{0}}\right)} \frac{1}{n^{3 / 2}}
$$

where $\zeta$ is a dimensionless number. It is reasonable to assume that $\zeta$ is of the order of unity since the dimensionless coefficient of $r(n) / m_{0}$ in a perturbative theory is supposed to be of the same order as the square of the coefficient of $f(n) / m_{0}$. We might also assume that $\zeta$ is purely real (and also negative), but this is not necessary because the imaginary part of $\zeta$ (if it exists) will not contribute to Eq. (5.12).

We shall now take into account $\mathbf{H}^{(2)}$ in the expression $[\mathbf{H},[\mathbf{H}, \mathbf{M}(0)]]$ in Eq. (5.10). The only term in $\langle[\mathbf{H},[\mathbf{H}, \mathbf{M}(0)]]\rangle$ which contains $\mathbf{H}^{(2)}$, and is of the order of $1 /\langle M(0)\rangle^{5}$, is $\left\langle\left[\mathbf{M}(0),\left[\mathbf{H}^{(2)}, \mathbf{M}(0)\right]\right]\right\rangle$. All the other terms are smaller by a factor of $1 /\langle M(0)\rangle^{2}$, and may thus be neglected. A straightforward calculation gives

$$
\left\langle\left[\mathbf{M}(0),\left[\mathbf{H}^{(2)}, \mathbf{M}(0)\right]\right]\right\rangle_{C T ; n_{0}}=2 \zeta \frac{(\hbar \varepsilon)^{2}}{\langle M(0)\rangle^{5}} e^{-\frac{\alpha}{8 n_{0}}} .
$$

Hence, we are now able to rewrite Eq. (5.12), including the second order correction of the Hamiltonian, as

$$
3 \mathrm{e}^{\left(\frac{\alpha}{8 n_{0}}\right)}+(1+4 \zeta) \mathrm{e}^{\left(-\frac{\alpha}{8 n_{0}}\right)}=4
$$

where $\zeta$ is still to be determined. Note that if we choose $\zeta=0$ (that is, $\mathbf{H}^{(2)}=0$ ) Eq. (B4) reduces to Eq. (5.12), as it was expected. Thus, we conclude that if $\alpha$ is of the order of unity, then Eq. (5.12) is satisfied automatically and we do not need a correction. This means that the Hamiltonian given in Eq. (3.3) with the interaction term (3.7) is equivalent to the exact Hamiltonian, at least up to $\mathrm{O}\left(\hbar^{2} /\langle M(0)\rangle^{3}\right)$. On the other hand, if $\alpha$ is $\mathrm{O}\left(n_{0}\right)$, then the interaction term (3.7) is only the first order term in a given perturbation theory.

In order to determine $\alpha$ in this last case, we use Eq. (B/4). There are two solutions of Eq. (B4), but only one of them is plausible:

$$
\mathrm{e}^{\left(\frac{\alpha}{8 n_{0}}\right)}=\frac{2+\sqrt{1-12 l}}{3}
$$

Note that $l \leq 0$ since $\alpha>0$ as it was expected. Relation (B5) does not determine $\alpha$, but instead express it as a function of the parameter $l$.

\section{APPENDIX C: SATURATION OF THE AREA-PHASE UNCERTAINTY RELATION}

We shall prove in this section that the CT-states defined in Eq. (3.20) saturate the area-phase uncertainty relation:

$$
\Delta \mathbf{N} \Delta \phi=\frac{1}{2}
$$

The variance of $\mathbf{N}$ for the Gaussian CT-states is given by

$$
\Delta \mathbf{N}=\sqrt{\frac{2 n_{0}}{\alpha}} .
$$


We now turn to calculate $\Delta \phi$.

First we define $\sin \phi$ in terms of the Susskind-Glogower's operators:

$$
\sin \phi=\frac{1}{2 i}\left(\mathbf{E}-\mathbf{E}^{\dagger}\right) .
$$

It is simple to verify that $\langle\sin \phi\rangle_{C T ; n_{0}}=0$; it is thus consistent to assume that also $\langle\phi\rangle_{C T ; n_{0}}=$ 0 , where $\phi$ has been chosen to have its eigenvalues in the interval $[-\pi, \pi]$. If $\alpha \sim 1$, the variance $\Delta \mathbf{N} \sim \sqrt{n_{0}}$ is quite large, which implies that $\Delta \phi$ is quite small. That is, $\phi$ is highly peaked around $\phi=0$. Hence we can assume that $\phi \approx \sin \phi$ which leads by Eq. (4.13) to

$$
\left\langle\phi^{2}\right\rangle_{C T ; n_{0}} \approx\left\langle(\sin \phi)^{2}\right\rangle_{C T ; n_{0}}=\frac{1}{2}\left(1-\exp \left(-\frac{\alpha}{4 n_{0}}\right)\right) \approx \frac{\alpha}{8 n_{0}}
$$

for $\alpha \sim 1$. Hence,

$$
\Delta \phi=\frac{1}{2} \sqrt{\frac{\alpha}{2 n_{0}}}
$$

and thus Eq. (C1) is satisfied.

For $\alpha \sim n_{0}$ it is impossible to assume that $\phi$ is highly peaked around zero; instead we shall assume that the main contribution to $\left\langle\phi^{2}\right\rangle_{C T ; n_{0}}$ comes from the region where $-\pi / 2<$ $\phi<\pi / 2$. In this interval $\phi$ can be expressed as:

$$
\phi^{2}=\lim _{m \rightarrow \infty}\left(\phi^{2}\right)_{m}
$$

where

$$
\left(\phi^{2}\right)_{m} \equiv \sum_{l=1}^{m} C_{l} \sin ^{2 l} \phi=\sin ^{2} \phi+\frac{1}{3} \sin ^{4} \phi+\frac{8}{45} \sin ^{6} \phi+\ldots+C_{m} \sin ^{2 m} \phi .
$$

The CT-states has the feature that

$$
\left\langle(\mathbf{E})^{l}\right\rangle_{C T ; n_{0}}=\left\langle\left(\mathbf{E}^{\dagger}\right)^{l}\right\rangle_{C T ; n_{0}}=\exp \left(-\frac{l^{2} \alpha^{*}}{16}\right)
$$

where $l$ is an integer and $\alpha^{*} \equiv \alpha / n_{0}$ is of the order of unity. Hence, expressing $\left(\phi^{2}\right)_{m}$ as polynomials in $\mathbf{E}$ and $\mathbf{E}^{\dagger}$ and then taking the average according to Eq. (C8), we were able to show (numerically) that

$$
\left\langle\left(\phi^{2}\right)_{m}\right\rangle_{C T ; n_{0}}=\frac{\alpha^{*}}{8}+\mathrm{O}\left(\left(\alpha^{*}\right)^{m+1}\right)
$$

were we have expressed the exponential functions in Eq. (C8) as a Taylor series of $\alpha^{*}$. Hence, we conclude that for $\alpha^{*}<1$

$$
\left\langle\phi^{2}\right\rangle_{C T ; n_{0}}=\frac{\alpha^{*}}{8}=\frac{\alpha}{8 n_{0}}
$$

which leads to Eq. (C1). 


\section{REFERENCES}

[1] K. Kuchař, gr-qc/9304012.

[2] S. Weinberg, in General Relativity: An Einstein Centenary Survey, eds. S. W. Hawking and W. Israel (Cambridge University Press, Cambridge 1979).

[3] M. B. Green, J. H. Schwarz and E. Witten, Superstring theory (Cambridge University Press, Cambridge 1987); J. Polchinski, String Theory (Cambridge University Press, Cambridge 1998).

[4] A. Ashtekar and K. Krasnov, gr-qc/9804039; A. Ashtekar, C. Rovelli and L. Smolin, Phys. Rev. Letters 69, 237 (1992).

[5] J. D. Bekenstein, Lett. Nuovo Cimento 11, 467 (1974).

[6] V. Mukhanov, Pis. Eksp. Teor. Fiz. 44, 50 (1986) [JETP Letters 44, 63 (1986)]

[7] J. D. Bekenstein and V. F. Mukhanov, Phys. Lett. B 360, 7 (1995).

[8] J. D. Bekenstein, gr-qc/9808028.

[9] D. Christodoulou, Phys. Rev. Lett. 25, 1596 (1970).

[10] S. W. Hawking, Phys. Rev. Lett. 26, 1344 (1971).

[11] R. Penrose and R. M. Floyd, Nature 229, 177 (1971).

[12] J. D. Bekenstein, Phys. Rev. D 7, 2333 (1973);

[13] J. D. Bekenstein, in Black Holes, Gravitational Radiation and the Universe, eds. B. R. Iyer and B. Bhawal (Kluwer, Dordrecht 1998).

[14] A. E. Mayo, Phys. Rev. D 58, 104007 (1998).

[15] M. Born, Atomic Physics (Blackie, London 1969), eight edition.

[16] S. Hod, Phys. Rev. D 59, 024014 (1999).

[17] S. W. Hawking, Commun. Math. Phys. 43, 199 (1975); Nature 248, 30 (1974).

[18] H. A. Kastrup, Phys. Lett. B 413, 267 (1997).

[19] C. Vaz and L. Witten, Phys. Lett. B 442, 90 (1998).

[20] J. Louko and J. Mäkelä, Phys. Rev. D 54, 4982 (1996).

[21] H. A. Kastrup, Phys. Lett. B 385, 75 (1996).

[22] S. Carlip and C. Teitelboim, Class. Quant. Grav. 12, 1699 (1995).

[23] R. Lynch, Phys. Rep. 256, 367 (1995).

[24] S. Hod, Phys. Rev. Lett. 81, 4293 (1998).

[25] D. N. Page, Phys. Rev. D 13, 198 (1976).

[26] J. J. Sakurai, Modern Quantum Mechanics (Addison Wesley Press, New York 1995).

[27] R. Ruffini and J. A. Wheeler, Physics Today 24, 30 (1971).

[28] M. Bojowald, H. A. Kastrup, F. Schramm and T. Strobl, gr-qc/9906105.

[29] S. W. Hawking, Phys. Rev. D 14, 2460 (1975).

[30] G. Gour, gr-qc/9907066 (To appear in Physical Review D).

[31] T. Padmanabhan, Phys. Lett. A 136, 203 (1989).

[32] H. C. Kim, M. H. Lee and J. Y. Ji, gr-qc/9709062.

[33] L. Susskind and J .Glogower, Physics 1, 49 (1964).

[34] S. Massar and R. Parentani, gr-qc/9903027.

[35] H. Umezawa, Advanced Field Theory (AIP Press, New York, 1995).

[36] J. D. Bekenstein, in Quantum Theory of Gravity, ed. S. M. Christensen (Adam Hilger, Bristol 1984).

[37] C. H. Wu and L. H. Ford, gr-qc/9905012. 